\title{
An Efficient, Clean, and Catalyst-Free Synthesis of Fused Pyrimidines Using Sonochemistry
}

\author{
M. Mamaghani, ${ }^{1}$ K. Tabatabaeian, ${ }^{1}$ R. Araghi, ${ }^{1}$ A. Fallah, ${ }^{2}$ and R. Hossein Nia ${ }^{1}$ \\ ${ }^{1}$ Department of Chemistry, Faculty of Sciences, University of Guilan, P.O. Box 41335-1914, Rasht, Iran \\ ${ }^{2}$ Department of Chemistry, Faculty of Sciences, Islamic Azad University, Rasht Branch, P.O. Box 41335-3516, Rasht, Iran \\ Correspondence should be addressed to M. Mamaghani; mchem41@gmail.com
}

Received 26 August 2014; Accepted 27 October 2014; Published 16 November 2014

Academic Editor: Jonathan White

Copyright (C) 2014 M. Mamaghani et al. This is an open access article distributed under the Creative Commons Attribution License, which permits unrestricted use, distribution, and reproduction in any medium, provided the original work is properly cited.

\begin{abstract}
In this report, synthesis of indenopyrido[2,3- $d]$ pyrimidine and pyrimido[4,5- $b]$ quinoline derivatives was investigated via onepot three-component reaction between 6-amino-2-(alkylthio)-pyrimidin-4(3H)one, 1,3-indanedione, or 1,3-cyclohexadione and arylaldehyde under ultrasonic irradiation in ethylene glycol as solvent at $65^{\circ} \mathrm{C}$. In these reactions fused pyrimidine derivatives were synthesized with high to excellent yields (82-97\%) and short reaction times (10-33 min).
\end{abstract}

\section{Introduction}

Pyrimidine structural moiety constitutes a major class of heterocyclic compounds which have various pharmaceutical applications. For example, they are found to possess antineoplastic [1-3], antiviral [4-6], antibiotic [7], and antiinflammatory properties [8]. Pyrimidines also exhibit a range of pharmacological activities such as antibacterial [9$11]$, antifungal $[12,13]$, anticancer [14, 15], and cardioprotective effects [16]. Bicyclic and tricyclic fused pyrimidine derivatives have received much attention in connection with biologically significant systems such as pyrido[2,3$d]$ pyrimidines. Pyrido[2,3- $d$ ] pyrimidine structural motif is present in pirenperone (tranquilizer) [17] and ramastine (antiallergic) [18], as well as in some antiulcerative and antiasthmatic agents [19]. In addition, quinolines have pharmacological properties which include wide applications in medicinal chemistry; for example, this scaffold structure is present in anti-inflammatory agents, antimalarial drugs, and antihypertensive, antiasthmatic, antibacterial, and tyrosine kinase inhibiting agents [20-25].

Moreover the importance of uracil and its annulated derivatives is well recognized by synthetic $[26,27]$ as well as biological $[28,29]$ chemists. The 6-amino-uracil derivatives represent very important classes of functionalized uracils; also 6-amino-uracils find wide applications as starting materials for the synthesis of a number of fused uracils of biological significance, for example, pyrano-, pyrido-, pyrazolo-, pyrimido-, and pyridazinopyrimidines $[30,31]$.

On the other hand, ultrasonic reactions have been increasingly used as clean, green, and environmentally benign routes for the preparation of organic compounds of synthetic and biological values [32-37]. A large number of organic reactions can be carried out in higher yield, shorter reaction time, and under milder conditions, by using ultrasonic irradiation [38-41].

These observations led us to attempt the synthesis of some new fused pyrimidine derivatives using 6-aminoalkyltiouracil as starting material under sonochemical conditions. The present work describes our approach for the synthesis of polyfunctional pyrimidines using green chemistry.

\section{Experimental}

2.1. Chemicals and Apparatus. Melting points were measured on an electrothermal 9100 apparatus. IR spectra were determined on a Shimadzo IR- 470 spectrometer. ${ }^{1} \mathrm{H}$ NMR and ${ }^{13} \mathrm{C}$ NMR spectra were recorded on a $400 \mathrm{MHz}$ Bruker DRX-400 in DMSO- $\mathrm{d}_{6}$ as solvent and TMS as an internal standard. Chemical shifts on ${ }^{1} \mathrm{H}$ and ${ }^{13} \mathrm{C}$ NMR were 
expressed in ppm downfield from tetramethylsilane. Sonication was performed in Elmasonic S $40 \mathrm{H}$ ultrasonic cleaning unit. Elemental analyses were carried out on a Carlo-Erba EA1110CNNO-S analyser and agreed with the calculated values. All the chemicals were purchased from Merck and used without further purification. All solvents used were dried and distilled according to standard procedures.

2.2. General Procedure for the Synthesis of $(4 a-r)$. A mixture of equimolar amounts of 6-amino-2-(alkylthio)pyrimidin$4(3 \mathrm{H})$-one 1 ( $1 \mathrm{mmol}), 1,3$-indanedione 2 ( $1 \mathrm{mmol})$, or 1,3cyclohexadione 5 and aldehydes 3 (1 mmol) in ethylene glycol $(5 \mathrm{~mL})$ was placed in a Pyrex-glass open vessel and irradiated at $65^{\circ} \mathrm{C}$ by ultrasonic irradiations $(40 \mathrm{kHz}$ ) (for conventional conditions, the reaction mixture was heated under reflux conditions). The progress of the reaction was monitored by TLC (EtOAc/petroleum ether 8:4). After completion of reaction, the mixture was concentrated and cooled. The solid obtained was filtered off and recrystallized from EtOH/ $\mathrm{H}_{2} \mathrm{O}$ $(1 / 1)$ to furnish the desired pure product.

\subsection{Spectral Data for the Products}

2.3.1. 5-(4-Chlorophenyl)-2-(methylthio)-3H-indeno[5, 6:1 $\left.1^{\prime}, 2^{\prime}\right]$ pyrido[2,3-d]pyrimidine-4,6(5H,11H)-dione (4a). Red Powder; M.p.: $>300^{\circ} \mathrm{C}$; IR (KBr) $v_{\max }=3448,3410,3271,3062$, 2932, 1680, 1653, 1585, 1541, 1502, 1452, 1350, 1271, 1078, 899, $742,707 \mathrm{~cm}^{-1} .{ }^{1} \mathrm{H}$ NMR $\left(400 \mathrm{MHz}, \mathrm{DMSO}-\mathrm{d}_{6}\right): \delta=2.60(\mathrm{~s}$, $\left.3 \mathrm{H}, \mathrm{CH}_{3}\right), 4.82(\mathrm{~s}, 1 \mathrm{H}, \mathrm{CH}), 7.30-7.25(\mathrm{~m}, 5 \mathrm{H}, \mathrm{Ar}-\mathrm{H}), 7.36$ $(\mathrm{t}, 1 \mathrm{H}, J=7.40 \mathrm{~Hz}, \mathrm{Ar}-\mathrm{H}), 7.45(\mathrm{t}, 1 \mathrm{H}, J=7.40 \mathrm{~Hz}, \mathrm{Ar}-\mathrm{H})$, 7.79 (d, $1 \mathrm{H}, J=7.20 \mathrm{~Hz}, \mathrm{Ar}-\mathrm{H}), 11.13$ (br s, $1 \mathrm{H}, \mathrm{NH}$ ), 12.60 (br s, $1 \mathrm{H}, \mathrm{CO}-\mathrm{NH}$ ) ppm. ${ }^{13} \mathrm{C}$ NMR $\left(100 \mathrm{MHz}, \mathrm{DMSO}-\mathrm{d}_{6}\right)$ : $\delta=13.3(\mathrm{Me}), 34.2(\mathrm{CH}), 99.9,107.9,120.4,120.9,128.4,130.1$, $130.8,131.2,132.4,133.6,136.6,137.5,144.8,153.5,155.8,161.5$ $(\mathrm{CONH}), 191.1(\mathrm{C}=\mathrm{O})$ ppm. Anal. Cald. for $\mathrm{C}_{21} \mathrm{H}_{14} \mathrm{ClN}_{3} \mathrm{O}_{2} \mathrm{~S}$ (407.87): C, 61.84; H, 3.46; N, 10.30\%; Found: C, 61.95; H, 3.31; $\mathrm{N}, 10.43 \%$.

2.3.2. 2-(Methylthio)-5-phenyl-3H-indeno[5,6:1',2']pyrido[2, 3-d]pyrimidine-4,6 $(5 \mathrm{H}, 11 \mathrm{H})$-dione $(\mathbf{4 b})$. Red Powder; M.p.: $>300^{\circ} \mathrm{C}$; IR $(\mathrm{KBr}) v_{\max }=3448,3040,2922,2851,1685,1647$, $1582,1537,1500,1456,1358,1273,742,702 \mathrm{~cm}^{-1} .{ }^{1} \mathrm{H}$ NMR $\left(400 \mathrm{MHz}, \mathrm{DMSO}-\mathrm{d}_{6}\right): \delta=2.60\left(\mathrm{~s}, 3 \mathrm{H}, \mathrm{CH}_{3}\right), 4.82(\mathrm{~s}, 1 \mathrm{H}$, $\mathrm{CH}), 7.15-7.11$ (m, 1H, Ar-H), 7.26-7.21 (m, 4H, Ar-H), 7.34 (t, $2 \mathrm{H}, J=7.40 \mathrm{~Hz}$, Ar-H), 7.44 (t, $1 \mathrm{H}, J=7.60 \mathrm{~Hz}$, Ar-H), 7.78 (d, $1 \mathrm{H}, J=7.20 \mathrm{~Hz}$, Ar-H), 11.07 (br s, $1 \mathrm{H}, \mathrm{NH}$ ), 12.55 (br s, $1 \mathrm{H}, \mathrm{CO}-\mathrm{NH}) \mathrm{ppm} .{ }^{13} \mathrm{CNMR}\left(100 \mathrm{MHz}, \mathrm{DMSO}-\mathrm{d}_{6}\right): \delta=$ $13.3(\mathrm{Me}), 34.5(\mathrm{CH}), 100.0,108.5,120.3,120.8,126.6,127.4$, $128.2,128.5,130.6,132.3,133.7,136.7,146.0,153.3,155.7,162.5$ $(\mathrm{CONH}), 191.1(\mathrm{C}=\mathrm{O}) \mathrm{ppm}$. Anal. Cald. for $\mathrm{C}_{21} \mathrm{H}_{15} \mathrm{~N}_{3} \mathrm{O}_{2} \mathrm{~S}$ : (373.43); C, 67.54; H, 4.05; N, 11.25\%; Found: C, 67.39; H, 4.12; $\mathrm{N}, 11.12 \%$.

2.3.3. 5-(4-Bromophenyl)-2-(methylthio)-3H-indeno $\left[5,6: 1^{\prime}, 2^{\prime}\right]$ pyrido[2,3-d]pyrimidine-4,6 $(5 \mathrm{H}, 11 \mathrm{H})$-dione $(4 \mathrm{c})$. Red Powder; M.p.: $>300^{\circ}$ C; IR (KBr): $\nu_{\max }=3380,3300,3100,3080$, 2920, 2820, 1690, 1640, 1618, 1558, 1498, 1440, 1348, 1270, 1070,
850, 800, $760 \mathrm{~cm}^{-1} \cdot{ }^{1} \mathrm{H}$ NMR $\left(400 \mathrm{MHz}, \mathrm{DMSO}-\mathrm{d}_{6}\right): \delta=2.60$ (s, 3H, $\left.\mathrm{CH}_{3}\right), 4.80$ (s, 1H, CH), 7.21 (d, 2H, J = 7.60 Hz, Ar-H), $7.26(\mathrm{~d}, 1 \mathrm{H}, J=6.80 \mathrm{~Hz}, \mathrm{Ar}-\mathrm{H}), 7.35$ (t, $1 \mathrm{H}, J=7.20 \mathrm{~Hz}, \mathrm{Ar}-\mathrm{H})$, 7.42 (m, 3H, Ar-H), 7.79 (d, $1 \mathrm{H}, J=6.80 \mathrm{~Hz}, \mathrm{Ar}-\mathrm{H}), 11.12$ (br s, $1 \mathrm{H}, \mathrm{NH}$ ), 12.58 (br s, $1 \mathrm{H}, \mathrm{CO}-\mathrm{NH}$ ) ppm. ${ }^{13} \mathrm{CNMR}$ $\left(100 \mathrm{MHz}, \mathrm{DMSO}-\mathrm{d}_{6}\right): \delta ; 13.3(\mathrm{Me}), 34.3(\mathrm{CH}), 99.9,107.8$, $119.7,120.4,120.9,129.6,130.5,130.8,131.3,132.4,133.6,136.6$, 145.2, 155.8, 162.1 (CONH), $191.1(\mathrm{C}=\mathrm{O}) \mathrm{ppm}$. Anal. Cald. for $\mathrm{C}_{21} \mathrm{H}_{14} \mathrm{BrN}_{3} \mathrm{O}_{2} \mathrm{~S}$ (452.32): C, 55.76; H, 3.12; N, 9.29\%; Found: C, 55.63; H, 3.01; N, 9.38\%.

\subsubsection{5-(4-Fluorophenyl)-2-(methylthio)-3H-indeno[5,6:1', $\left.2^{\prime}\right]$} pyrido[2,3-d]pyrimidine-4,6 $(5 \mathrm{H}, 11 \mathrm{H})$-dione $(4 d)$. Red Powder; M.p.: $>300^{\circ} \mathrm{C}$; IR (KBr): $\nu_{\max }=3440,3400,3026,2921$, 2840, 1677, 1641, 1602, 1578, 1539, 1493, 1458, 1357, 1275, 1149, $843,798,748,709 \mathrm{~cm}^{-1} .{ }^{1} \mathrm{H}$ NMR $\left(400 \mathrm{MHz}\right.$, DMSO-d $\left.\mathrm{d}_{6}\right): \delta=$ 2.60 (s, 3H, $\mathrm{CH}_{3}$ ), 4.83 (s, 1H, CH), 7.07-7.01 (m, 4H, Ar-H), $7.26(\mathrm{~d}, 2 \mathrm{H}, J=6.40 \mathrm{~Hz}, \mathrm{Ar}-\mathrm{H}), 7.35$ (t, $1 \mathrm{H}, J=7.40 \mathrm{~Hz}, \mathrm{Ar}-\mathrm{H})$, 7.45 (t, $1 \mathrm{H}, J=7.20 \mathrm{~Hz}, \mathrm{Ar}-\mathrm{H}), 7.79$ (d, $1 \mathrm{H}, J=7.20 \mathrm{~Hz}, \mathrm{Ar}-\mathrm{H})$, 11.10 (br s, 1H, NH), 12.56 (br s, $1 \mathrm{H}, \mathrm{CO}-\mathrm{NH}$ ) ppm. ${ }^{13} \mathrm{C} \mathrm{NMR}$ $\left(100 \mathrm{MHz}, \mathrm{DMSO}-\mathrm{d}_{6}\right): \delta=13.3(\mathrm{Me}), 34.0(\mathrm{CH}), 99.6,108.1$, $114.9\left(\mathrm{~d},{ }^{2} J_{\mathrm{C}-\mathrm{F}}=21.0 \mathrm{~Hz}\right), 120.3,120.7,129.9\left(\mathrm{~d},{ }^{3} J_{\mathrm{C}-\mathrm{F}}=\right.$ 8.0 Hz), 130.6, 132.1, 133.9, 136.8, 142.6, 153.3, 156.1, 158.9, 160.6 $\left(\mathrm{d},{ }^{1} J_{\mathrm{C}-\mathrm{F}}=240.0 \mathrm{~Hz}\right), 191.0(\mathrm{C}=\mathrm{O}) \mathrm{ppm}$. Anal. Cald. for $\mathrm{C}_{21} \mathrm{H}_{14} \mathrm{FN}_{3} \mathrm{O}_{2} \mathrm{~S}$ (391.42): C, 64.44; H, 3.61; N, 10.74\%; Found: C, $64.38 ; \mathrm{H}, 3.62 ; \mathrm{N}, 10.85 \%$.

2.3.5. 5-(2-Bromophenyl)-2-(methylthio)-3H-indeno[5, 6:1 $\left.1^{\prime}, 2^{\prime}\right]$ pyrido[2,3-d]pyrimidine-4,6 $(5 \mathrm{H}, 11 \mathrm{H})$-dione $(4 \mathrm{e})$. Red Powder; M.p.: $>300^{\circ} \mathrm{C}$; IR (KBr): $\nu_{\max }=3350,3300,3110,3080$, 2920, 2820, 1690, 1645, 1618, 1558, 1498, 1440, 1347, 1270, 1070, $895,845,800,760,710 \mathrm{~cm}^{-1}$. ${ }^{1} \mathrm{H}$ NMR $(400 \mathrm{MHz}, \mathrm{DMSO}-$ $\left.\mathrm{d}_{6}\right): \delta=2.60\left(\mathrm{~s}, 3 \mathrm{H}, \mathrm{CH}_{3}\right), 5.24(\mathrm{~s}, 1 \mathrm{H}, \mathrm{CH}), 7.06(\mathrm{dt}, 1 \mathrm{H}, J=$ $8.40 \mathrm{~Hz}, 2.2 \mathrm{~Hz}, \mathrm{Ar}-\mathrm{H}), 7.28-7.21$ (m, 3H, Ar-H), 7.35 (t, 1H, $J=$ $7.40 \mathrm{~Hz}, \mathrm{Ar}-\mathrm{H}), 7.48-7.43(\mathrm{~m}, 2 \mathrm{H}, \mathrm{Ar}-\mathrm{H}), 7.80$ (d, 1H, J = $7.20 \mathrm{~Hz}, \mathrm{Ar}-\mathrm{H}$ ), 11.11 (br s, $1 \mathrm{H}, \mathrm{NH}$ ), 12.26 (br s, $1 \mathrm{H}, \mathrm{CO}-$ $\mathrm{NH})$, ppm. ${ }^{13} \mathrm{C}$ NMR $\left(100 \mathrm{MHz}, \mathrm{DMSO}-\mathrm{d}_{6}\right): \delta=13.3(\mathrm{Me})$, $35.7(\mathrm{CH}), 100.0,107.8,120.4,120.7,123.4,128.1,128.4,130.7$, $132.0,132.2,132.8,133.6,136.6,136.7,144.9,153.7,155.6,162.1$ $(\mathrm{CONH}), 190.7(\mathrm{C}=\mathrm{O})$ ppm. Anal. Cald. for $\mathrm{C}_{21} \mathrm{H}_{14} \mathrm{BrN}_{3} \mathrm{O}_{2} \mathrm{~S}$ (452.32): C, 55.76; H, 3.12; N, 9.29\%. Found: C, 55.83; H, 3.08; N, $9.45 \%$.

2.3.6. 5-(2-Chlorophenyl)-2-(methylthio)-3H-indeno $\left[5,6: 1^{\prime}, 2^{\prime}\right]$ pyrido[2,3-d]pyrimidine-4,6 $(5 \mathrm{H}, 11 \mathrm{H})$-dione $(4 \mathrm{f})$. Red Powder; M.p.: $>300^{\circ} \mathrm{C}$; IR (KBr): $v_{\max }=3460,3385,3120,3060$, 2930, 2854, 1685, 1649, 1616, 1580, 1541, 1502, 1445, 1345, 1277, $1078,893,764 \mathrm{~cm}^{-1} .{ }^{1} \mathrm{H}$ NMR $\left(400 \mathrm{MHz}, \mathrm{DMSO}-\mathrm{d}_{6}\right): \delta=2.60$ (s, 3H, $\mathrm{CH}_{3}$ ), 5.24 (s, 1H, CH), 7.15 (d, 1H, J = 7.20 Hz, Ar-H), $7.21(\mathrm{t}, 2 \mathrm{H}, J=7.40 \mathrm{~Hz}, \mathrm{Ar}-\mathrm{H}), 7.29$ (d, 2H, $J=7.20 \mathrm{~Hz}, \mathrm{Ar}-\mathrm{H})$, 7.35 (t, 1H, $J=7.20 \mathrm{~Hz}, \mathrm{Ar}-\mathrm{H}), 7.44(\mathrm{t}, 1 \mathrm{H}, J=7.20 \mathrm{~Hz}, \mathrm{Ar}-\mathrm{H})$, 7.79 (d, $1 \mathrm{H}, J=7.20 \mathrm{~Hz}, \mathrm{Ar}-\mathrm{H}$ ), 11.10 (br s, 1H, NH), 12.49 (br s, $1 \mathrm{H}, \mathrm{CO}-\mathrm{NH}) \mathrm{ppm} .{ }^{13} \mathrm{CNMR}\left(100 \mathrm{MHz}, \mathrm{DMSO}-\mathrm{d}_{6}\right): \delta=$ $13.3(\mathrm{Me}), 33.3(\mathrm{CH}), 99.9,107.6,120.4,120.7,127.4,128.1,129.5$, $130.7,131.9,132.2,132.9,133.6,136.6,143.1,153.7,155.9,162.1$ $(\mathrm{CONH}), 190.7(\mathrm{C}=\mathrm{O})$ ppm. Anal. Cald. for $\mathrm{C}_{21} \mathrm{H}_{14} \mathrm{ClN}_{3} \mathrm{O}_{2} \mathrm{~S}$ (407.87): C, 61.84; H, 3.46; N, 10.30; Found: C, 61.72; H, 3.31; $\mathrm{N}, 10.18 \%$. 
2.3.7. 5-(2-Hydroxyphenyl)-2-(methylthio)-3H-indeno [5, 6:1', $\left.2^{\prime}\right]$ pyrido $[2,3-d]$ pyrimidine-4,6 $(5 \mathrm{H}, 11 \mathrm{H})$-dione $(\mathbf{4 g})$. Orang Powder; M.p.: $>300^{\circ} \mathrm{C}$; IR $(\mathrm{KBr}): v_{\max }=3450,3270,3050$, $2920,2820,1700,1645,1620,1600,1560,1500,1440,1347$, $1280,1180,868,800,760,700 \mathrm{~cm}^{-1} .{ }^{1} \mathrm{H} N M R(400 \mathrm{MHz}$, DMSO- $\left.\mathrm{d}_{6}\right): \delta=2.59\left(\mathrm{~s}, 3 \mathrm{H}, \mathrm{CH}_{3}\right), 4.98(\mathrm{~s}, 1 \mathrm{H}, \mathrm{CH}), 6.72-6.69$ (m, 2H, Ar-H), 7.03-7.0 (m, 2H, Ar-H), $7.25(\mathrm{~d}, 1 \mathrm{H}, J=$ $6.80 \mathrm{~Hz}, \mathrm{Ar}-\mathrm{H}), 7.35(\mathrm{t}, 1 \mathrm{H}, J=7.40 \mathrm{~Hz}, \mathrm{Ar}-\mathrm{H}), 7.45(\mathrm{t}, 1 \mathrm{H}, J=$ $7.40 \mathrm{~Hz}, \mathrm{Ar}-\mathrm{H}$ ), 7.79 (d, $1 \mathrm{H}, J=7.20 \mathrm{~Hz}, \mathrm{Ar}-\mathrm{H}), 9.58$ (br s, $1 \mathrm{H}, \mathrm{NH}$ ), 11.12 (s, 1H, OH), 12.73 (br s, $1 \mathrm{H}, \mathrm{CO}-\mathrm{NH}$ ) ppm. ${ }^{13} \mathrm{C}$ NMR $(100 \mathrm{MHz}$, DMSO-d $\mathrm{d}): \delta=13.3(\mathrm{Me}), 29.6(\mathrm{CH})$, $100.0,107.9,117.5,120.0,120.2,120.8,127.9,130.0,130.6,132.3$, $133.8,136.8,136.9,154.8,155.0,156.4,156.5,163.9(\mathrm{CONH})$, 191.1 (C=O) ppm. Anal. Cald. for $\mathrm{C}_{21} \mathrm{H}_{15} \mathrm{~N}_{3} \mathrm{O}_{3} \mathrm{~S}$ (389.43): C, 64.77; H, 3.88; N, 10.79\%; Found: C, 64.70; H, 3.98; N, $10.88 \%$.

2.3.8. 5-(2,4-Dichlorophenyl)-2-(methylthio)-3H-indeno[5,

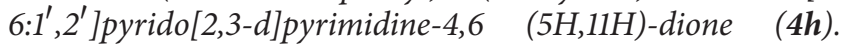
Red Powder; M.p.: $>300^{\circ} \mathrm{C}$; IR (KBr): $v_{\max }=3448,3228$, $3066,2940,2854,1683,1649,1580,1539,1506,1460,1352$, $1275,1103,1060,858,764,704 \mathrm{~cm}^{-1} .{ }^{1} \mathrm{H}$ NMR $(400 \mathrm{MHz}$, DMSO-d $\left.\mathrm{d}_{6}\right): \delta=2.60\left(\mathrm{~s}, 3 \mathrm{H}, \mathrm{CH}_{3}\right), 5.22(\mathrm{~s}, 1 \mathrm{H}, \mathrm{CH}), 7.23(\mathrm{~d}$, $1 \mathrm{H}, J=6.40 \mathrm{~Hz}$, Ar-H), 7.37-7.27 (m, 3H, Ar-H), 7.47-7.44 (m, 2H, Ar-H), 7.80 (d, $1 \mathrm{H}, J=7.20 \mathrm{~Hz}, \mathrm{Ar}-\mathrm{H}), 11.13$ (br s, $1 \mathrm{H}$, $\mathrm{NH}), 12.52$ (br s, $1 \mathrm{H}, \mathrm{CO}-\mathrm{NH}) \mathrm{ppm} .{ }^{13} \mathrm{C}$ NMR $(100 \mathrm{MHz}$, DMSO- $\left.\mathrm{d}_{6}\right): \delta=13.3(\mathrm{Me}), 33.0(\mathrm{CH}), 99.4,107.1,120.5,120.7$, $127.6,128.7,130.8,131.7,132.2,133.0,133.5,133.9,142.3,136.6$, 153.8, 156.0, $162.0(\mathrm{CONH}), 190.7(\mathrm{C}=\mathrm{O}) \mathrm{ppm}$. Anal. Cald. for $\mathrm{C}_{21} \mathrm{H}_{13} \mathrm{Cl}_{2} \mathrm{~N}_{3} \mathrm{O}_{2} \mathrm{~S}$ : (442.32) C, 57.02; $\mathrm{H}, 2.96 ; \mathrm{N}, 9.50 \%$; Found: C, 56.88; H, 2.83; N, 9.35\%.

2.3.9. 5-(2-Nitrophenyl)-2-(methylthio)-3H-indeno[5,6:1', $\left.2^{\prime}\right]$ pyrido[2,3-d]pyrimidine-4,6 (5H,11H)-dione (4i). Red Powder; M.p.: $>300^{\circ} \mathrm{C}$; IR $(\mathrm{KBr}): \nu_{\max }=3350,3060,2923$, $1685,1649,1578,1540,1504,1450,1539,1342,1277,770$, $708 \mathrm{~cm}^{-1} .{ }^{1} \mathrm{H}$ NMR $\left(400 \mathrm{MHz}, \mathrm{DMSO}-\mathrm{d}_{6}\right): \delta=2.60(\mathrm{~s}, 3 \mathrm{H}$, $\left.\mathrm{CH}_{3}\right), 4.83(\mathrm{~s}, 1 \mathrm{H}, \mathrm{CH}), 7.05(\mathrm{t}, 2 \mathrm{H}, J=8.80 \mathrm{~Hz}, \mathrm{Ar}-\mathrm{H})$, 7.29-7.25 (m, 3H, Ar-H), 7.35 (t, 1H, $J=7.40 \mathrm{~Hz}$, Ar-H), 7.45 (t, $1 \mathrm{H}, J=7.40 \mathrm{~Hz}, \mathrm{Ar}-\mathrm{H}), 7.79$ (d, $1 \mathrm{H}, J=7.20 \mathrm{~Hz}, \mathrm{Ar}-\mathrm{H})$, 11.10 (br s, $1 \mathrm{H}, \mathrm{NH}), 12.57$ (br s, $1 \mathrm{H}, \mathrm{CO}-\mathrm{NH}$ ) ppm. ${ }^{13} \mathrm{C}$ NMR $\left(100 \mathrm{MHz}, \mathrm{DMSO}-\mathrm{d}_{6}\right): \delta=13.3(\mathrm{Me}), 33.9(\mathrm{CH}), 97.0,108.2$, 115.0, 115.2, 120.4, 120.9, 126.3, 129.9, 130.0, 130.8, 132.4, 133.6, 136.7, 142.1, 155.7, 160.0, $162.4(\mathrm{CONH}), 191.1(\mathrm{C}=\mathrm{O}) \mathrm{ppm}$. Anal. Cald. for $\mathrm{C}_{21} \mathrm{H}_{14} \mathrm{~N}_{4} \mathrm{O}_{4} \mathrm{~S}$ (418.43) C, 60.28; H, 3.37; N, 13.39\%; Found: C, 60.10; H, 3.48; N, 13.51\%.

2.3.10. 5-(4-Methoxyphenyl)-2-(methylthio)-3H-indeno[5,6:1', $2^{\prime}$ ]pyrido[2,3-d]pyrimidine-4,6 (5H,11H)-dione (4j). Red Powder; M.p.: $>300^{\circ} \mathrm{C}$; IR $(\mathrm{KBr}): v_{\max }=3211,3040,2920$, $2851,1674,1645,1606,1554,1500,1442,1352,1261,1219,1020$, $898,835,766,706 \mathrm{~cm}^{-1} .{ }^{1} \mathrm{H}$ NMR $\left(400 \mathrm{MHz}, \mathrm{DMSO}-\mathrm{d}_{6}\right): \delta=$ 2.59 (s, 3H, $\left.\mathrm{CH}_{3}\right), 3.68\left(\mathrm{~s}, 3 \mathrm{H}, \mathrm{OCH}_{3}\right), 4.76(\mathrm{~s}, 1 \mathrm{H}, \mathrm{CH}), 6.79$ $(\mathrm{d}, 2 \mathrm{H}, J=8.20 \mathrm{~Hz}, \mathrm{Ar}-\mathrm{H}), 7.14(\mathrm{~d}, 2 \mathrm{H}, J=8.20 \mathrm{~Hz}, \mathrm{Ar}-\mathrm{H})$, 7.26 (d, $1 \mathrm{H}, J=7.20 \mathrm{~Hz}, \mathrm{Ar}-\mathrm{H}), 7.35$ (t, $1 \mathrm{H}, J=7.20 \mathrm{~Hz}, \mathrm{Ar}-\mathrm{H}$ ), $7.44(\mathrm{t}, 1 \mathrm{H}, J=7.40 \mathrm{~Hz}, \mathrm{Ar}-\mathrm{H}), 7.78(\mathrm{~d}, 1 \mathrm{H}, J=6.80 \mathrm{~Hz}, \mathrm{Ar}-\mathrm{H})$, 11.03 (br s, $1 \mathrm{H}, \mathrm{NH}$ ), 12.53 (br s, $1 \mathrm{H}, \mathrm{CO}-\mathrm{NH}$ ) ppm. ${ }^{13} \mathrm{C} \mathrm{NMR}$ $\left(100 \mathrm{MHz}, \mathrm{DMSO}-\mathrm{d}_{6}\right): \delta=13.3(\mathrm{Me}), 33.6(\mathrm{CH}), 55.4(\mathrm{MeO})$,
95.0, 108.7, 112.8, 113.9, 120.2, 120.8, 129.1, 130.6, 132.3, 133.7, 136.8, 138.2, 155.4, 158.1, $161.5(\mathrm{CONH}), 191.2(\mathrm{C}=\mathrm{O}) \mathrm{ppm}$. Anal. Cald. for $\mathrm{C}_{22} \mathrm{H}_{17} \mathrm{~N}_{3} \mathrm{O}_{3} \mathrm{~S}$ : (403.45) C, 65.49; H, 4.25; N, 10.42\%; Found: C, 65.30; H, 4.35; N, 10.28\%.

2.3.11. 2-(Methylthio)-5-(p-tolyl)-3H-indeno[5,6:1',2' $2^{\prime}$ pyrido [2,3-d]pyrimidine-4,6 $(5 \mathrm{H}, 11 \mathrm{H})$-dione $(\mathbf{4 k})$. Red Powder; M.p.: $>300^{\circ} \mathrm{C}$; IR (KBr): $v_{\max }=3490,3227,3059,2940,2854$, $1681,1645,1585,1539,1504,1456,1350,1275,897,773 \mathrm{~cm}^{-1}$. ${ }^{1} \mathrm{H}$ NMR $\left(400 \mathrm{MHz}\right.$, DMSO-d $\left.{ }_{6}\right): \delta=2.21\left(\mathrm{~s}, 3 \mathrm{H}, \mathrm{CH}_{3}\right), 2.59$ (s, $\left.3 \mathrm{H}, \mathrm{CH}_{3}\right), 4.77(\mathrm{~s}, 1 \mathrm{H}, \mathrm{CH}), 7.03(\mathrm{~d}, 2 \mathrm{H}, J=7.20 \mathrm{~Hz}, \mathrm{Ar}-\mathrm{H})$, $7.12(\mathrm{~d}, 2 \mathrm{H}, J=7.20 \mathrm{~Hz}, \mathrm{Ar}-\mathrm{H}), 7.25$ (d, $1 \mathrm{H}, J=6.40 \mathrm{~Hz}, \mathrm{Ar}-\mathrm{H})$, $7.34(\mathrm{t}, 1 \mathrm{H}, J=7.20 \mathrm{~Hz}, \mathrm{Ar}-\mathrm{H}), 7.44(\mathrm{t}, 1 \mathrm{H}, J=7.20 \mathrm{~Hz}, \mathrm{Ar}-\mathrm{H})$, $7.77(\mathrm{~d}, 1 \mathrm{H}, J=6.80 \mathrm{~Hz}, \mathrm{Ar}-\mathrm{H}), 11.04$ (br s, $1 \mathrm{H}, \mathrm{NH}), 12.54$ (br s, $1 \mathrm{H}, \mathrm{CO}-\mathrm{NH}$ ) ppm. ${ }^{13} \mathrm{C}$ NMR $\left(100 \mathrm{MHz}, \mathrm{DMSO}-\mathrm{d}_{6}\right)$ : $\delta=13.3(\mathrm{Me}), 21.0(\mathrm{Me}), 34.1(\mathrm{CH}), 100.2,108.7,120.2,120.8$, $128.0,128.4,129.0,130.6,132.3,133.7,135.6,136.8,143.1,153.2$, 155.5, $162.4(\mathrm{CONH}), 191.1(\mathrm{C}=\mathrm{O}) \mathrm{ppm}$. Anal. Cald. for $\mathrm{C}_{22} \mathrm{H}_{17} \mathrm{~N}_{3} \mathrm{O}_{2} \mathrm{~S}$ : (387.45) C, 68.20; H, 4.42; N, 10.85\%; Found: C, $68.38 ; \mathrm{H}, 4.25 ; \mathrm{N}, 10.78 \%$.

2.3.12. 5-(3,4-Dimethoxyphenyl)-2-(methylthio)-3H-indeno[5, 6:1', $\left.2^{\prime}\right]$ pyrido[2,3-d]pyrimidine-4,6 (5H,11H)-dione $(4 \mathrm{l})$. Red Powder; M.p.: $>300^{\circ} \mathrm{C}$; IR $(\mathrm{KBr}): v_{\max }=3389$, $3223,3130,3063,2933,2839,1691,1653,1554,1504$, 1450, 1340, 1226, 1024, 897, 798, 764, $710 \mathrm{~cm}^{-1} .{ }^{1} \mathrm{HNMR}$ $\left(400 \mathrm{MHz}, \mathrm{DMSO}-\mathrm{d}_{6}\right): \delta=2.60\left(\mathrm{~s}, 3 \mathrm{H}, \mathrm{SCH}_{3}\right), 3.67$ (s, $\left.3 \mathrm{H}, \mathrm{OCH}_{3}\right), 3.71\left(\mathrm{~s}, 3 \mathrm{H}, \mathrm{OCH}_{3}\right), 4.79(\mathrm{~s}, 1 \mathrm{H}, \mathrm{CH})$, $6.64(\mathrm{~d}, 1 \mathrm{H}, J=8.40 \mathrm{~Hz}, \operatorname{Ar}-\mathrm{H}), 6.74(\mathrm{~d}, 1 \mathrm{H}, J=$ $8.40 \mathrm{~Hz}, \mathrm{Ar}-\mathrm{H}), 6.95(\mathrm{~s}, 1 \mathrm{H}, \mathrm{Ar}-\mathrm{H}), 7.27(\mathrm{~d}, 1 \mathrm{H}, J=6.80 \mathrm{~Hz}$, Ar-H), $7.34(\mathrm{t}, 1 \mathrm{H}, J=7.20 \mathrm{~Hz}, \mathrm{Ar}-\mathrm{H}), 7.43(\mathrm{t}, 1 \mathrm{H}, J=7.20 \mathrm{~Hz}$, Ar-H), 7.77 (d, $1 \mathrm{H}, J=7.20 \mathrm{~Hz}, \operatorname{Ar}-\mathrm{H}), 11.01$ (br s, $1 \mathrm{H}, \mathrm{NH}$ ), 12.54 (br s, $1 \mathrm{H}, \mathrm{CO}-\mathrm{NH}) \mathrm{ppm} .{ }^{13} \mathrm{C}$ NMR (100 MHz, DMSO$\left.\mathrm{d}_{6}\right): \delta=13.3(\mathrm{Me}), 33.9(\mathrm{CH}), 55.9(\mathrm{MeO}), 56.0(\mathrm{MeO}), 100$, $108.5,112.3,112.6,119.8,120.2,120.8,130.6,132.3,133.7,136.7$, 138.6, 147.8, 148.6, 155.4, $162.5(\mathrm{CONH}), 191.2(\mathrm{C}=\mathrm{O}) \mathrm{ppm}$. Anal. Cald. for $\mathrm{C}_{23} \mathrm{H}_{19} \mathrm{~N}_{3} \mathrm{O}_{4} \mathrm{~S}$ : (433.48) C, 63.73; H, 4.42; N, 9.69\%; Found: C, 63.88; H, 4.33; N, 9.86\%.

2.3.13. 5-(2,4-Dichlorophenyl)-2-(ethylthio)-5,11-dihydro-3Hindeno $\left[2^{\prime}, 1^{\prime}: 5,6\right]$ pyrido $[2,3$-d]pyrimidine-4,6-dione (4m). Red Powder; M.p.: $282-284^{\circ} \mathrm{C}$; IR (KBr): $v_{\max }=3203$, 3136, 3059, 2966, 2926, 1649, 1607, 1550, 1502, $14501350,1190,1101$, $1070,858,764,700 \mathrm{~cm}^{-1} .{ }^{1} \mathrm{H}$ NMR $(400 \mathrm{MHz}$, DMSO-d 6 ): $\delta=$ $1.36\left(\mathrm{t}, 3 \mathrm{H}, J=7.1 \mathrm{~Hz}, \mathrm{CH}_{3}\right), 3.22\left(\mathrm{q}, 2 \mathrm{H}, J=7.1 \mathrm{~Hz}, \mathrm{CH}_{2}\right), 5.22$ (s, $1 \mathrm{H}, \mathrm{CH}), 7.22(\mathrm{~d}, 1 \mathrm{H}, J=6.8 \mathrm{~Hz}, \mathrm{Ar}-\mathrm{H}), 7.46-7.26(\mathrm{~m}, 5 \mathrm{H}$, Ar-H), 7.80 (d, 1H, J = 7.2 Hz, Ar-H), 11.09 (s, 1H, NH), 12.48 (br s, $1 \mathrm{H}, \mathrm{NH}-\mathrm{C}=\mathrm{O}) \mathrm{ppm} .{ }^{13} \mathrm{C}$ NMR $\left(100 \mathrm{MHz}, \mathrm{DMSO}-\mathrm{d}_{6}\right)$ : $\delta=15.0(\mathrm{Me}), 24.7\left(\mathrm{CH}_{2}\right), 33.0(\mathrm{CH}), 99.4,107.1,120.5,127.5$, 127.6, 128.7, 130.8, 131.7, 132.2, 133.0, 133.5, 133.9, 136.6, 142.3, 153.8, 156.0, $162.0(\mathrm{CONH}), 190.7(\mathrm{C}=\mathrm{O}) \mathrm{ppm}$. Anal. Cald. for $\mathrm{C}_{22} \mathrm{H}_{15} \mathrm{Cl}_{2} \mathrm{~N}_{3} \mathrm{O}_{2} \mathrm{~S}$ (456.34): C, 57.90; H, 3.31; N, 9.21\%; Found: C, 57.74; H, 3.23; N, 9.05\%.

2.3.14. 5-(4-Chlorophenyl)-2-(ethylthio)-5,11-dihydro-3H-indeno $\left[2^{\prime}, 1^{\prime}: 5,6\right]$ pyrido[2,3-d]pyrimidine-4,6-dione $(\mathbf{4 n})$. Red Powder; M.p.: $268-270^{\circ} \mathrm{C}$, IR (KBr): $v_{\max }=3209,3140$, $3063,2876,1649,1545,1504,1452,1350,1188,1146,860,764$, $704 \mathrm{~cm}^{-1} .{ }^{1} \mathrm{H}$ NMR $\left(400 \mathrm{MHz}\right.$, DMSO-d $\left.\mathrm{d}_{6}\right): \delta=1.36(\mathrm{t}, 3 \mathrm{H}$, 
$\left.J=7.2 \mathrm{~Hz}, \mathrm{CH}_{3}\right), 3.23\left(\mathrm{q}, 2 \mathrm{H}, J=7.2 \mathrm{~Hz}, \mathrm{CH}_{2}\right), 4.82(\mathrm{~s}, 1 \mathrm{H}$, $\mathrm{CH}), 7.26$ (m, 5H, Ar-H), 7.35 (t, 1H, J = 7.2 Hz, Ar-H), 7.45 (t, $1 \mathrm{H}, J=7.4 \mathrm{~Hz}, \mathrm{Ar}-\mathrm{H}), 7.79$ (d, $1 \mathrm{H}, J=7.2 \mathrm{~Hz}, \mathrm{Ar}-\mathrm{H}), 11.08$ (s, 1H, NH), 12.56 (br s, $1 \mathrm{H}, \mathrm{NH}-\mathrm{C}=\mathrm{O}$ ) ppm. ${ }^{13} \mathrm{C} \mathrm{NMR}$ $\left(100 \mathrm{MHz}, \mathrm{DMSO}-\mathrm{d}_{6}\right): \delta=15.1(\mathrm{Me}), 24.6\left(\mathrm{CH}_{2}\right), 34.2(\mathrm{CH})$, $99.9,112.0,107.9,120.4,120.9,128.4,130.1,130.8,131.2,132.3$, $133.5,136.6,144.8,155.8,191.1(\mathrm{C}=\mathrm{O}) \mathrm{ppm}$. Anal. Cald. for $\mathrm{C}_{22} \mathrm{H}_{16} \mathrm{ClN}_{3} \mathrm{O}_{2} \mathrm{~S}$ (421.90): C, 62.63; H, 3.82; N, 9.96\%; Found: C, 62.38; H, 3.95; N, 9.76\%.

2.3.15. 2-(Ethylthio)-5-(2-fluorophenyl)-5,11-dihydro-3H-indeno $\left[2^{\prime}, 1^{\prime}: 5,6\right]$ pyrido $[2,3-d]$ pyrimidine-4,6-dione (4o). Red Powder; M.p.: $257-259^{\circ} \mathrm{C}$; IR $(\mathrm{KBr}): v_{\max }=3221,3132$, 3057, 2932, 1684, 1647, 1583, 1539, 1491, 1350, 1263, 1182, 760, $706 \mathrm{~cm}^{-1}$. ${ }^{1} \mathrm{H}$ NMR $\left(400 \mathrm{MHz}, \mathrm{DMSO}-\mathrm{d}_{6}\right): \delta=1.37(\mathrm{t}, 3 \mathrm{H}$, $\left.J=7.2 \mathrm{~Hz}, \mathrm{CH}_{3}\right), 3.23\left(\mathrm{q}, 2 \mathrm{H}, J=7.2 \mathrm{~Hz}, \mathrm{CH}_{2}\right), 5.04(\mathrm{~s}, 1 \mathrm{H}$, $\mathrm{CH}), 7.03$ (m, 2H, Ar-H), $7.18(\mathrm{~m}, 1 \mathrm{H}, \mathrm{Ar}-\mathrm{H}), 7.25$ (t, 2H, Ar-H), 7.34 (t, 1H, J = 7.2 Hz, Ar-H), $7.44(\mathrm{t}, 1 \mathrm{H}, J=7.2 \mathrm{~Hz}$, Ar-H), 7.80 (d, $1 \mathrm{H}, J=6.8 \mathrm{~Hz}, \operatorname{Ar}-\mathrm{H}), 11.06$ (s, 1H, NH), 12.48 (br s, $1 \mathrm{H}, \mathrm{NH}-\mathrm{C}=\mathrm{O})$ ppm. ${ }^{13} \mathrm{C} \mathrm{NMR}\left(100 \mathrm{MHz}, \mathrm{DMSO}-\mathrm{d}_{6}\right)$ : $\delta=15.1(\mathrm{Me}), 24.6\left(\mathrm{CH}_{2}\right), 29.0(\mathrm{CH}), 99.4,107.4,115.5\left({ }^{2} \mathrm{~J}=\right.$ $22.0 \mathrm{~Hz}), 120.4\left({ }^{3} \mathrm{~J}=10.0 \mathrm{~Hz}\right), 120.8,124.6,128.4,128.5,130.7$, $131.1,132.2,132.8,132.9,133.6,136.7,153.6,155.8$ (CONH), $160.3\left({ }^{1} J_{\mathrm{C}-\mathrm{F}}=245.0 \mathrm{~Hz}\right), 191.0(\mathrm{C}=\mathrm{O}) \mathrm{ppm}$. Anal. Cald. for $\mathrm{C}_{22} \mathrm{H}_{16} \mathrm{FN}_{3} \mathrm{O}_{2} \mathrm{~S}$ (405.44): C, 65.17; H, 3.98; N, 10.36\%; Found: C, $65.06 ; \mathrm{H}, 3.81 ; \mathrm{N}, 10.22 \%$.

2.3.16. 5-(4-Bromophenyl)-2-(ethylthio)-5,11-dihydro-3H-indeno $\left[2^{\prime}, 1^{\prime}: 5,6\right]$ pyrido $[2,3-d]$ pyrimidine-4,6-dione (4p). Red Powder; M.p.: $255-257^{\circ} \mathrm{C}$, IR (KBr): $\nu_{\max }=3230,2858$, $1645,1578,1539,1499,1450,1352,1182,1016,797,708 \mathrm{~cm}^{-1}$. ${ }^{1} \mathrm{H}$ NMR $\left(400 \mathrm{MHz}\right.$, DMSO- $\left.\mathrm{d}_{6}\right): \delta=1.36(\mathrm{t}, 3 \mathrm{H}, J=7.2 \mathrm{~Hz}$, $\left.\mathrm{CH}_{3}\right), 3.23\left(\mathrm{q}, 2 \mathrm{H}, J=7.2 \mathrm{~Hz}, \mathrm{CH}_{2}\right), 4.81(\mathrm{~s}, 1 \mathrm{H}, \mathrm{CH}), 7.21(\mathrm{~d}$, $2 \mathrm{H}, J=8.0 \mathrm{~Hz}$, Ar-H), $7.26(\mathrm{~d}, 1 \mathrm{H}, J=7.2 \mathrm{~Hz}, \mathrm{Ar}-\mathrm{H}), 7.35$ (t, $1 \mathrm{H}, J=7.2 \mathrm{~Hz}, \mathrm{Ar}-\mathrm{H}), 7.44(\mathrm{~m}, 3 \mathrm{H}, \mathrm{Ar}-\mathrm{H}), 7.79$ (d, 1H, $J=$ $7.2 \mathrm{~Hz}, \mathrm{Ar}-\mathrm{H}$ ), 11.07 (s, 1H, NH), 12.55 (br s, 1H, NH-C=O) ppm; ${ }^{13}$ C NMR (100 MHz, DMSO-d 6 ): $\delta=15.1(\mathrm{Me}), 24.6$ $\left(\mathrm{CH}_{2}\right), 34.4(\mathrm{CH}), 99.7,107.8,119.7,120.4,120.9,130.5,130.7$, $131.3,132.3,133.6,136.6,145.2,155.8,161.8$ (CONH), 191.0 $(\mathrm{C}=\mathrm{O})$ ppm. Anal. Cald. for $\mathrm{C}_{22} \mathrm{H}_{16} \mathrm{BrN}_{3} \mathrm{O}_{2} \mathrm{~S}$ (466.35): C, 56.66; H, 3.46; N, 9.01\%; Found: C, 56.53; H, 3.27; N, 9.16\%.

2.3.17. 2-(Ethylthio)-5-(4-methylthiophenyl)-5,11-dihydro-3Hindeno $\left[2^{\prime}, 1^{\prime}: 5,6\right]$ pyrido[2,3-d]pyrimidine-4,6-dione (4q). Red Powder; M.p.: 250-252 ${ }^{\circ}$ C; IR (KBr) $\nu_{\max }=3219,3138$, 3057, 2964, 2922, 1645, 1551, 1499, 1450, 1354, 1184, 835, 768, $712 \mathrm{~cm}^{-1}$. ${ }^{1} \mathrm{H}$ NMR $\left(400 \mathrm{MHz}, \mathrm{DMSO}-\mathrm{d}_{6}\right): \delta=1.36(\mathrm{t}, 3 \mathrm{H}$, $\left.J=7.2 \mathrm{~Hz}, \mathrm{CH}_{3}\right), 2.41\left(\mathrm{~s}, 3 \mathrm{H}, \mathrm{CH}_{3}\right), 3.22(\mathrm{q}, 2 \mathrm{H}, J=7.2 \mathrm{~Hz}$, $\mathrm{CH}_{2}$ ), 4.78 (s, $\left.1 \mathrm{H}, \mathrm{CH}\right), 7.13(\mathrm{~d}, 2 \mathrm{H}, J=8.0 \mathrm{~Hz}, \mathrm{Ar}-\mathrm{H}), 7.19$ $(\mathrm{d}, 2 \mathrm{H}, J=8.0 \mathrm{~Hz}, \mathrm{Ar}-\mathrm{H}), 7.26(\mathrm{~d}, 1 \mathrm{H}, J=7.2 \mathrm{~Hz}, \mathrm{Ar}-\mathrm{H})$, $7.34(\mathrm{t}, 1 \mathrm{H}, J=7.2 \mathrm{~Hz}, \mathrm{Ar}-\mathrm{H}), 7.44$ (t, $1 \mathrm{H}, J=7.4 \mathrm{~Hz}, \mathrm{Ar}-\mathrm{H})$, 7.78 (d, 1H, J = 7.2 Hz, Ar-H), 11.03 (s, 1H, NH), 12.53 (br s, $1 \mathrm{H}, \mathrm{NH}-\mathrm{C}=\mathrm{O})$ ppm. ${ }^{13} \mathrm{C}$ NMR $\left(100 \mathrm{MHz}, \mathrm{DMSO}-\mathrm{d}_{6}\right): \delta=$ $15.1(\mathrm{Me}), 15.4(\mathrm{Me}), 24.7\left(\mathrm{CH}_{2}\right), 34.1(\mathrm{CH}), 100.0,108.3$, $120.3,120.8,126.4,128.8,130.6,132.3,133.7,136.0,136.7,142.9$, $153.8,154.0,155.5,155.6,191.1(\mathrm{C}=\mathrm{O}) \mathrm{ppm}$. Anal. Cald. for $\mathrm{C}_{23} \mathrm{H}_{19} \mathrm{~N}_{3} \mathrm{O}_{2} \mathrm{~S}_{2}$ (433.55): C, 63.72; H, 4.42; N, 9.69\%; Found: C, 63.57 ; H, 4.59 ; N, 9.56\%.
2.3.18. 2-(Ethylthio)-5-phenyl-5,11-dihydro-3H-indeno $\left[2^{\prime}, 1^{\prime}: 5\right.$, 6]pyrido[2,3-d]pyrimidine-4,6-dione (4r). Red Powder; M.p.: 273-276 C; IR (KBr): $\nu_{\max }=3119,3026,2922,2849,1716$, $1645,1541,1495,1452,1352,1184,744,710 \mathrm{~cm}^{-1} .{ }^{1} \mathrm{H}$ NMR $\left(400 \mathrm{MHz}, \mathrm{DMSO}-\mathrm{d}_{6}\right): \delta=1.36\left(\mathrm{t}, 3 \mathrm{H}, J=7.2 \mathrm{~Hz}, \mathrm{CH}_{3}\right), 3.23$ $\left(\mathrm{q}, 2 \mathrm{H}, J=7.2 \mathrm{~Hz}, \mathrm{CH}_{2}\right), 4.82(\mathrm{~s}, 1 \mathrm{H}, \mathrm{CH}), 7.13(\mathrm{~m}, 1 \mathrm{H}, \mathrm{Ar}-\mathrm{H})$, 7.24 (m, 5H, Ar-H), 7.35 (t, 1H, J = 7.4 Hz, Ar-H), 7.44 (t, 1H, J= $7.4 \mathrm{~Hz}, \mathrm{Ar}-\mathrm{H}), 7.79$ (d, 1H, J = 7.2 Hz, Ar-H), 11.02 (s, 1H, NH), 12.52 (br s, $1 \mathrm{H}, \mathrm{NH}-\mathrm{C}=\mathrm{O}$ ) ppm. ${ }^{13} \mathrm{C}$ NMR $(100 \mathrm{MHz}, \mathrm{DMSO}-$ $\left.\mathrm{d}_{6}\right): \delta=15.1(\mathrm{Me}), 24.6\left(\mathrm{CH}_{2}\right), 34.5(\mathrm{CH}), 108.5,120.3,120.9$, $126.6,128.2,128.5,130.7,132.3,133.6,136.6,145.9,155.6,162.5$ $(\mathrm{CONH}), 191.2(\mathrm{C}=\mathrm{O})$ ppm. Anal. Cald. for $\mathrm{C}_{22} \mathrm{H}_{17} \mathrm{~N}_{3} \mathrm{O}_{2} \mathrm{~S}$ (387.10): C, 68.26; H, 4.42; N, 10.85\%; Found: C, 68.08; H, 4.55; N, $10.97 \%$.

2.3.19. 2-(Ethylthio)-5-(4-fluorophenyl)-5,8,9,10-tetrahydro3H,7H-pyrimido[4,5-b]quinoline-4,6-dione (6a). White Powder; M.p.: 300-302 ${ }^{\circ}$ C; IR (KBr): $\nu_{\max }=3221,3142,3067$, 3032, 2957, 2880, 1647, 1607, 1553, 1483, 1364, 1226, 1186, 837, $797 \mathrm{~cm}^{-1} .{ }^{1} \mathrm{H}$ NMR $(400 \mathrm{MHz}$, DMSO-d 6 ): $\delta=1.31(\mathrm{t}, 3 \mathrm{H}, J=$ $7.2 \mathrm{~Hz}, \mathrm{CH}_{3}$ ), 1.95-1.82 (m, 2H, $\left.\mathrm{CH}_{2}-\mathrm{CH}_{2}-\mathrm{CH}_{2}\right), 2.29-2.18$ (m, $2 \mathrm{H}, \mathrm{CH}_{2}-\mathrm{CH}_{2}-$ ), 2.66-2.56 (m, $2 \mathrm{H}, \mathrm{CH}_{2}-\mathrm{CH}_{2}-\mathrm{C}=\mathrm{O}$ ), $3.13\left(\mathrm{q}, 2 \mathrm{H}, \mathrm{J}=7.2 \mathrm{~Hz}, \mathrm{CH}_{2}-\mathrm{CH}_{3}\right), 4.95(\mathrm{~s}, 1 \mathrm{H}, \overline{\mathrm{CH}}), 7.01(\mathrm{t}$, $2 \mathrm{H}, J=8.8 \mathrm{~Hz}, \mathrm{Ar}-\mathrm{H}), 7.22(\mathrm{dd}, 2 \mathrm{H}, J=8.2,5.8 \mathrm{~Hz}, \mathrm{Ar}-\mathrm{H})$, 9.85 (s, 1H, NH), 12.33 (br s, $1 \mathrm{H}, \mathrm{NH}-\mathrm{C}=\mathrm{O}$ ) ppm. ${ }^{13} \mathrm{CNMR}$ $\left(100 \mathrm{MHz}, \mathrm{DMSO}-\mathrm{d}_{6}\right): \delta=15.1(\mathrm{Me}), 21.3\left(\mathrm{CH}_{2}\right), 24.5\left(\mathrm{CH}_{2}\right)$, $26.9\left(\mathrm{CH}_{2}\right), 33.2\left(\mathrm{CH}_{2}-\mathrm{C}=\mathrm{O}\right), 37.2(\mathrm{CH}), 98.4,110.9,115.0$ $\left({ }^{2} J_{\mathrm{CF}}=21.0 \mathrm{~Hz}\right), 129.6\left({ }^{3} J_{\mathrm{CF}}=8.0 \mathrm{~Hz}\right), 143.3\left({ }^{4} J_{\mathrm{CF}}=2.0 \mathrm{~Hz}\right)$, $152.4,153.5,153.6,161.0\left({ }^{1} J_{\mathrm{CF}}=240.0 \mathrm{~Hz}\right), 162.2(\mathrm{CONH})$, $195.0(\mathrm{C}=\mathrm{O})$ ppm. Anal. Cald. for $\mathrm{C}_{19} \mathrm{H}_{18} \mathrm{FN}_{3} \mathrm{O}_{2} \mathrm{~S}$ (371.43): C, 61.44; H, 4.88; N, 11.31\%; Found: C, 61.32; H, 4.70; N, 11.18\%.

2.3.20. 2-(Ethylthio)-5-(naphthalen-2-yl)-5,8,9,10-tetrahydro$3 \mathrm{H}, 7 \mathrm{H}$-pyrimido[4,5-b]quinoline-4,6-dione (6b). White Powder; M.p.: $310-312^{\circ} \mathrm{C}$; IR (KBr): $\nu_{\max }=3221,3138,3063$, 3028, 2953, 2874, 1647, 1607, 1554, 1479, 1450, 1366, 1186, 797, $752 \mathrm{~cm}^{-1} .{ }^{1} \mathrm{H}$ NMR $\left(400 \mathrm{MHz}, \mathrm{DMSO}-\mathrm{d}_{6}\right): \delta=1.31(\mathrm{t}, 3 \mathrm{H}, J=$ $\left.7.2 \mathrm{~Hz}, \mathrm{CH}_{3}\right), 1.94-1.85\left(2 \mathrm{H}, \mathrm{m}, \mathrm{CH}_{2}-\mathrm{CH}_{2}-\mathrm{CH}_{2}\right), 2.31-2.18$ (m, 2H, $\underline{\mathrm{CH}}_{2}-\mathrm{CH}_{2}-$ ), 2.70-2.60 (m, 2 $\left.\mathrm{H}, \overline{\mathrm{C}} \mathrm{H}_{2}-\mathrm{CH}_{2}-\mathrm{C}=\mathrm{O}\right)$, 3.15 (q, $\left.2 \mathrm{H}, \bar{J}=7.2 \mathrm{~Hz}, \mathrm{CH}_{2}-\mathrm{CH}_{3}\right), 5.12$ (s, $\left.1 \mathrm{H}, \mathrm{CH}\right), 7.43$

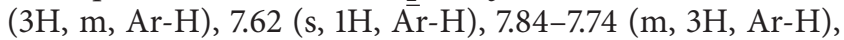
9.88 (s, 1H, NH), 12.33 (br s, $1 \mathrm{H}, \mathrm{NH}-\mathrm{C}=\mathrm{O}$ ) ppm. ${ }^{13} \mathrm{C} \mathrm{NMR}$ $\left(100 \mathrm{MHz}, \mathrm{DMSO}-\mathrm{d}_{6}\right): \delta=15.1(\mathrm{Me}), 21.3\left(\mathrm{CH}_{2}\right), 24.5\left(\mathrm{CH}_{2}\right)$, $26.9\left(\mathrm{CH}_{2}\right), 27.0\left(\mathrm{CH}_{2}-\mathrm{C}=\mathrm{O}\right), 34.2(\mathrm{CH}), 98.3,111.0,125.7$, $125.8,126.2,127.7,127.2,127.9,128.2$, 132.2, 133.2, 144.6, 153.5, 153.6, $194.9(\mathrm{C}=\mathrm{O})$ ppm. Anal. Cald. for $\mathrm{C}_{23} \mathrm{H}_{21} \mathrm{~N}_{3} \mathrm{O}_{2} \mathrm{~S}$ (403.50): C, 68.46; H, 5.25; N, 10.41\%; Found: C, 68.60; H, $5.17 ; \mathrm{N}, 10.55 \%$.

2.3.21. 2-(Ethylthio)-5-(p-tolyl)-5,8,9,10-tetrahydro-3H,7Hpyrimido[4,5-b]quinoline-4,6-dione (6c). White Powder; M.p.: $292-294^{\circ} \mathrm{C}$; IR (KBr): $v_{\max }=3225,3142,3067$, 2878, 1649, 1614, 1556, 1475, 1366, 1186, $794 \mathrm{~cm}^{-1} \cdot{ }^{1} \mathrm{H} \mathrm{NMR}$ $\left(400 \mathrm{MHz}, \mathrm{DMSO}-\mathrm{d}_{6}\right): \delta=1.31\left(\mathrm{t}, 3 \mathrm{H}, J=7.2 \mathrm{~Hz}, \mathrm{CH}_{3}-\right.$ $\left.\mathrm{CH}_{2}\right), 1.92-1.80\left(\mathrm{~m}, 2 \mathrm{H}, \mathrm{CH}_{2}-\mathrm{CH}_{2}-\mathrm{CH}_{2}\right), 2.3-2.1(\mathrm{~m}, 5 \overline{\mathrm{H}}$, $\underline{\mathrm{CH}}_{2}-\mathrm{CH}_{2}-$ and $\left.\mathrm{CH}_{3}-\mathrm{Ar}\right), 2.65-2 . \overline{5} 7\left(\mathrm{~m}, 2 \mathrm{H}, \mathrm{CH}_{2}-\mathrm{CH}_{2}-\right.$ $\mathrm{C}=\overline{\mathrm{O}}$ ), 3.14 (q, $\left.2 \mathrm{H}, \mathrm{J}=7.2 \mathrm{~Hz}, \mathrm{CH}_{2}-\mathrm{CH}_{3}\right), 4.90$ (s, $1 \mathrm{H}, \mathrm{CH}$ ), 6.99 (d, $2 \mathrm{H}, J=7.6 \mathrm{~Hz}, \mathrm{Ar}-\mathrm{H}), 7.08$ (d, $2 \mathrm{H}, J=7.6 \mathrm{~Hz}, \mathrm{Ar}-\mathrm{H})$, 


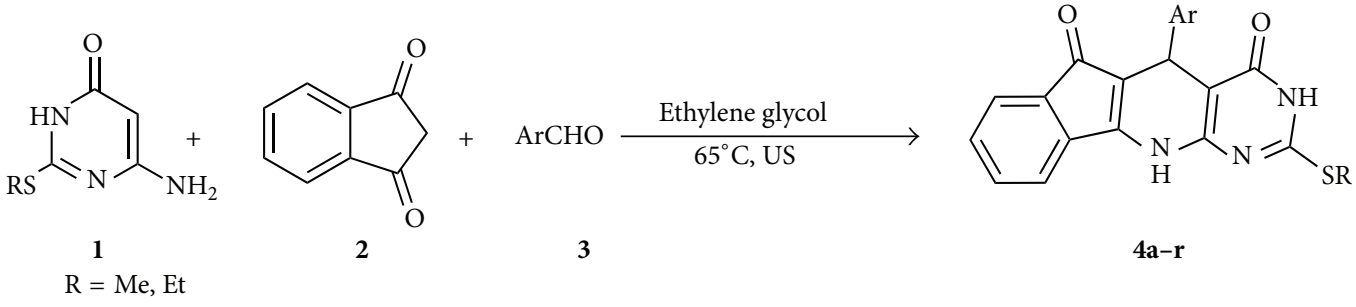

Scheme 1: Synthesis of indenopyrido[2,3-d]pyrimidine (4a-r).<smiles>CCc1nc(N)cc(=O)[nH]1</smiles>

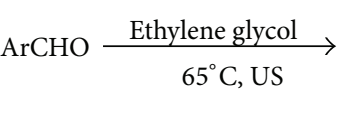

3<smiles>CCc1nc2c(c(=O)[nH]1)C(C#N)C1=C(CCCC1=O)N2</smiles>

Scheme 2: Synthesis of of pyrimido[4,5-b]quinoline $\mathbf{6 a - f}$.

9.79 (s, 1H, NH), 12.31 (br s, $1 \mathrm{H}, \mathrm{NH}-\mathrm{C}=\mathrm{O}$ ) ppm. ${ }^{13} \mathrm{C} \mathrm{NMR}$ $\left(100 \mathrm{MHz}, \mathrm{DMSO}-\mathrm{d}_{6}\right): \delta=15.1(\mathrm{Me}), 21.0(\mathrm{Me}), 21.3\left(\mathrm{CH}_{2}\right)$, $24.5\left(\mathrm{CH}_{2}\right), 26.8\left(\mathrm{CH}_{2}\right), 26.9\left(\mathrm{CH}_{2}-\mathrm{C}=\mathrm{O}\right), 33.3(\mathrm{CH}), 98.6$, 111.2, 127.8, 128.9, 135.2, 144.2, 153.2, 153.3, $194.8(\mathrm{C}=\mathrm{O}) \mathrm{ppm}$. Anal. Cald. for $\mathrm{C}_{20} \mathrm{H}_{21} \mathrm{~N}_{3} \mathrm{O}_{2} \mathrm{~S}$ (367.46): C, 65.37; H, 5.76; N, 11.41\%; Found: C, 65.21; H, 5.52; N, 11.25\%.

2.3.22. 2-(Ethylthio)-5-(4-methoxyphenyl)-5,8,9,10-tetrahydro-3H,7H-pyrimido[4,5-b]quinoline-4,6-dione (6d). White Powder; M.p.: $288-290^{\circ} \mathrm{C}$; IR (KBr): $\nu_{\max }=3225,3140,3067$, 3030, 2949, 1649, 1608, 1553, 1479, 1366, 1184, 1230, 1034, $835 \mathrm{~cm}^{-1}$. ${ }^{1} \mathrm{H}$ NMR $\left(400 \mathrm{MHz}, \mathrm{DMSO}-\mathrm{d}_{6}\right): \delta=1.31(\mathrm{t}, 3 \mathrm{H}$, $\left.J=7.2 \mathrm{~Hz}, \mathrm{CH}_{3}\right), 1.95-1.81\left(\mathrm{~m}, 2 \mathrm{H}, \mathrm{CH}_{2}-\mathrm{CH}_{2}-\mathrm{CH}_{2}\right), 2.23$ (m, 2H, $\mathrm{CH}_{2}-\mathrm{CH}_{2}-$ ), 2.60-2.55 (m, 2H, $\mathrm{CH}_{2}-\mathrm{CH}_{2}-\mathrm{C}=\mathrm{O}$ ), $3.13\left(\mathrm{q}, 2 \mathrm{H}, \mathrm{J}=\overline{7.2 \mathrm{~Hz}}, \mathrm{CH}_{2}-\mathrm{CH}_{3}\right), 3.67$ (s, 3H, $\mathrm{OCH}_{3}$ ),

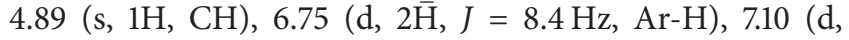
$2 \mathrm{H}, J=8.4 \mathrm{~Hz}, \operatorname{Ar}-\mathrm{H}$ ), 9.78 (s, $1 \mathrm{H}, \mathrm{NH}$ ), 12.30 (br s, $1 \mathrm{H}$, $\mathrm{NH}-\mathrm{C}=\mathrm{O}) \mathrm{ppm} .{ }^{13} \mathrm{CNMR}\left(100 \mathrm{MHz}, \mathrm{DMSO}-\mathrm{d}_{6}\right): \delta=15.1$ (Me), $21.3(\mathrm{MeO}), 24.5\left(\mathrm{CH}_{2}\right), 26.9\left(\mathrm{CH}_{2}\right), 32.8\left(\mathrm{CH}_{2}\right), 37.2$ $\left(\underline{\mathrm{CH}}_{2}-\mathrm{C}=\mathrm{O}\right), 55.4(\mathrm{CH}), 98.8,111.3,113.7,128.8,139.5,153.1$, 153.2, 157.9, $162.0(\mathrm{CONH}), 194.8(\mathrm{C}=\mathrm{O}) \mathrm{ppm}$. Anal. Cald. for $\mathrm{C}_{22} \mathrm{H}_{21} \mathrm{~N}_{3} \mathrm{O}_{2} \mathrm{~S}$ (383.46): C, 62.64; H, 5.52; N, 10.96\%; Found: C, $62.64 ; \mathrm{H}, 5.58 ; \mathrm{N}, 1085 \%$.

2.3.23. 2-(Ethylthio)-5-(5-methylthiophen-2-yl)-5,8,9,10-tetrahydro-3H,7H-pyrimido[4,5-b]quinoline-4,6-dione (6e). White Powder; M.p.: $280-282^{\circ} \mathrm{C}$; IR $(\mathrm{KBr}): v_{\max }=3223$, 3138, 3067, 3030, 2951, 2872, 1649, 1607, 1553, 1479, 1448, 1364, 1182, $798 \mathrm{~cm}^{-1}$. ${ }^{1} \mathrm{HNMR}\left(400 \mathrm{MHz}, \mathrm{DMSO}-\mathrm{d}_{6}\right): \delta=$ $1.32\left(\mathrm{t}, 3 \mathrm{H}, J=7.2 \mathrm{~Hz}, \mathrm{CH}_{3}\right), 1.98-1.85\left(\mathrm{~m}, 2 \mathrm{H}, \mathrm{CH}_{2}-\mathrm{CH}_{2}-\right.$ $\left.\mathrm{CH}_{2}-\right)$, 2.27 (m, 2H, $\left.\mathrm{CH}_{2}-\mathrm{CH}_{2}-\right), 2.30$ (s, 3H, $\left.\mathrm{CH}_{3}-\mathrm{A} \overline{\mathrm{r}}\right)$, 2.62-2.54 (m, 2H, $\left.\mathrm{CH}_{2}-\underline{\mathrm{CH}}_{2}-\mathrm{C}=\mathrm{O}\right), 3.13(\mathrm{q}, 2 \mathrm{H}, J=7.2 \mathrm{~Hz}$, $\left.\mathrm{CH}_{2}-\mathrm{CH}_{3}\right), 5.15$ (s, $\left.1 \mathrm{H}, \widehat{\mathrm{CH}}\right), 6.48$ (s, 2H, Ar-H), 9.90 (s, $1 \mathrm{H}$, $\mathrm{NH}), 12.41$ (br s, $1 \mathrm{H}, \mathrm{NH}-\mathrm{C}=\mathrm{O}$ ) ppm. ${ }^{13} \mathrm{C} \mathrm{NMR}(100 \mathrm{MHz}$, DMSO-d $\left.\mathrm{d}_{6}\right): \delta=15.1(\mathrm{Me}), 15.4(\mathrm{Me}), 21.3\left(\mathrm{CH}_{2}\right), 24.6\left(\mathrm{CH}_{2}\right)$, $26.9\left(\mathrm{CH}_{2}\right), 28.6\left(\mathrm{CH}_{2}-\mathrm{C}=\mathrm{O}\right), 37.2(\mathrm{CH}), 98.0,110.6,123.0$, $125.0,137.0,148.6,152.5,153.4,162.0(\mathrm{CONH}), 194.7(\mathrm{C}=\mathrm{O})$ ppm. Anal. Cald. for $\mathrm{C}_{18} \mathrm{H}_{19} \mathrm{~N}_{3} \mathrm{O}_{2} \mathrm{~S}_{2}$ (373.09): C, 57.88; $\mathrm{H}$, 5.13 ; N, 11.25\%; Found: C, 57.95; H, 5.20; N, 11.10\%.

2.3.24. 5-(4-Chlorophenyl)-2-(ethylthio)-5,8,9,10-tetrahydro$3 \mathrm{H}, 7 \mathrm{H}$-pyrimido[4,5-b]quinoline-4,6-dione (6f). White Powder; M.p.: $262-265^{\circ} \mathrm{C}$; IR (KBr) $v_{\max }=3261,3182,3045$, 2941, 2822, 1647, 1553, 1500, 1454, 1342, 1188, 1063, $825 \mathrm{~cm}^{-1}$. ${ }^{1} \mathrm{H} \mathrm{NMR}\left(400 \mathrm{MHz}, \mathrm{DMSO}-\mathrm{d}_{6}\right): \delta=1.31(\mathrm{t}, 3 \mathrm{H}, J=7.2 \mathrm{~Hz}$, $\left.\mathrm{CH}_{3}\right), 1.95-1.82\left(\mathrm{~m}, 2 \mathrm{H}, \mathrm{CH}_{2}-\mathrm{CH}_{2}-\mathrm{CH}_{2}\right), 2.25-2.21(\mathrm{~m}, 2 \mathrm{H}$, $\mathrm{CH}_{2}-\underline{\mathrm{CH}}_{2}-$ ), 2.65-2.55 (m, 2. $\mathrm{H}, \overline{\mathrm{C}} \mathrm{H}_{2}-\underline{\mathrm{CH}}_{2}-\mathrm{C}=\mathrm{O}$ ), 3.13 (q, $\left.2 \mathrm{H}, J=7.2 \mathrm{~Hz}, \mathrm{CH}_{2}-\mathrm{CH}_{3}\right), 4.92(\mathrm{~s}, 1 \mathrm{H}, \mathrm{CH}), 7.21(\mathrm{~d}, 2 \mathrm{H}, J=$ $8.4 \mathrm{~Hz}, \mathrm{Ar}-\mathrm{H}), 7.25^{-}$(d, $\left.2 \mathrm{H}, J=8.4 \mathrm{~Hz}, \mathrm{Ar}-\mathrm{H}\right), 9.86$ (s, $1 \mathrm{H}$, $\mathrm{NH}), 12.36$ (br s, $1 \mathrm{H}, \mathrm{NH}-\mathrm{C}=\mathrm{O}$ ) ppm. ${ }^{13} \mathrm{C} \mathrm{NMR}(100 \mathrm{MHz}$, DMSO-d $\left.\mathrm{d}_{6}\right): \delta=15.1(\mathrm{Me}), 21.2\left(\mathrm{CH}_{2}\right), 24.6\left(\mathrm{CH}_{2}\right), 26.8$ $\left(\mathrm{CH}_{2}\right), 33.6\left(\mathrm{CH}_{2}-\mathrm{C}=\mathrm{O}\right), 37.1(\mathrm{CH}), 98.1,110.7,110.7,128.2$, 129.8, 130.8, 131.6, 146.0, 153.6, 153.7, 194.9 (C=O) ppm. Anal. Cald. for $\mathrm{C}_{19} \mathrm{H}_{18} \mathrm{ClN}_{3} \mathrm{O}_{2} \mathrm{~S}$ (387.89): C, 58.83; H, 4.68; N, 10.83\%; Found: C, 58.70; H, 4.47; N, 10.68\%.

\section{Results and Discussion}

As part of our continuing efforts on the development of new synthetic strategies for the preparation of heterocyclic compounds [42-48], herein we wish to report one-pot threecomponent synthesis of fused pyridopyrimidines under sonochemical conditions without the use of catalyst (Schemes 1 and 2).

In the initial experiment, to optimize the reaction conditions, different solvents were screened for the synthesis of 5-(4-chlorophenyl)-2-(methylthio)-3H-indeno $\left[5,6: 1^{\prime}, 2^{\prime}\right]$ pyrido $[2,3-d]$ pyrimidine- $4,6(5 H, 11 H)$-dione $4 \mathbf{a}$ as a model reaction. The reaction between equimolar amounts of 6-amino-2-(methylthio)pyrimidin-4(3H)-one 1 (prepared by the condensation of thiourea with ethylcyanoacetate in sodium ethoxide and followed by alkylation with alkyliodide [49]), 1,3-indanedione 2, and 4-chlorobenzaldehyde were examined in various solvents and different temperatures 
TABLE 1: Effect of various solvents in the synthesis of $\mathbf{4 a}$ under conventional conditions and ultrasonic irradiations.

\begin{tabular}{|c|c|c|c|c|c|}
\hline \multirow{2}{*}{ Entry } & \multirow{2}{*}{ Solvent } & \multicolumn{2}{|c|}{ Conventional method } & \multicolumn{2}{|c|}{ Sonochemical method } \\
\hline & & Time (min) & Yield $(\%)^{\mathrm{a}}$ & Time (min) & Yield $(\%)^{\mathrm{a}}$ \\
\hline 1 & Ethylene glycol & 180 & $90^{\mathrm{b}}$ & 25 & $95^{\mathrm{c}}$ \\
\hline 2 & $\mathrm{AcOH}$ & 180 & 86 & 29 & 90 \\
\hline 3 & DMF & 210 & 82 & 29 & 88 \\
\hline 4 & $\mathrm{EtOH}$ & 220 & 84 & 30 & 88 \\
\hline 5 & $\mathrm{H}_{2} \mathrm{O}$ & 240 & 78 & 41 & 83 \\
\hline 6 & Acetone & 240 & 70 & 42 & 76 \\
\hline 7 & $\mathrm{CHCl}_{3}$ & 270 & 66 & 47 & 70 \\
\hline 8 & $\mathrm{CH}_{3} \mathrm{CN}$ & 300 & 60 & 50 & 65 \\
\hline
\end{tabular}

${ }^{\mathrm{a}}$ Isolated yield.

${ }^{\mathrm{b}}$ Reflux temperature.

${ }^{\mathrm{c}} 65^{\circ} \mathrm{C}$.

TABLE 2: Synthesis of indenopyrido[2,3-d]pyrimidine $\mathbf{4 a - r}$.

\begin{tabular}{|c|c|c|c|c|c|}
\hline Entry & Product & $\mathrm{R}$ & $\mathrm{Ar}$ & Time (min) & Yield $(\%)^{a}$ \\
\hline 1 & $4 a$ & $\mathrm{Me}$ & $4-\mathrm{ClC}_{6} \mathrm{H}_{4}$ & 25 & 95 \\
\hline 2 & $4 b$ & $\mathrm{Me}$ & $\mathrm{C}_{6} \mathrm{H}_{5}$ & 33 & 92 \\
\hline 3 & $4 c$ & $\mathrm{Me}$ & $4-\mathrm{BrC}_{6} \mathrm{H}_{4}$ & $28(220)^{\mathrm{b}}$ & $95(91)^{\mathrm{b}}$ \\
\hline 4 & $4 d$ & $\mathrm{Me}$ & $4-\mathrm{FC}_{6} \mathrm{H}_{4}$ & $20(160)^{\mathrm{b}}$ & $97(85)^{\mathrm{b}}$ \\
\hline 5 & $4 e$ & $\mathrm{Me}$ & $2-\mathrm{BrC}_{6} \mathrm{H}_{4}$ & $30(220)^{\mathrm{b}}$ & $94(88)^{\mathrm{b}}$ \\
\hline 6 & $4 \mathrm{f}$ & $\mathrm{Me}$ & $2-\mathrm{ClC}_{6} \mathrm{H}_{4}$ & $27(210)^{\mathrm{b}}$ & $96(89)^{\mathrm{b}}$ \\
\hline 7 & $4 \mathrm{~g}$ & $\mathrm{Me}$ & $2-\mathrm{OHC}_{6} \mathrm{H}_{4}$ & $32(240)^{\mathrm{b}}$ & $90(90)^{b}$ \\
\hline 8 & $4 h$ & $\mathrm{Me}$ & $2,4-\mathrm{Cl}_{2} \mathrm{C}_{6} \mathrm{H}_{3}$ & $20(160)^{b}$ & $96(90)^{b}$ \\
\hline 9 & $4 i$ & $\mathrm{Me}$ & $2-\mathrm{NO}_{2} \mathrm{C}_{6} \mathrm{H}_{4}$ & $22(170)^{\mathrm{b}}$ & $97(90)^{\mathrm{b}}$ \\
\hline 10 & $4 \mathrm{~g}$ & $\mathrm{Me}$ & $4-\mathrm{OCH}_{3} \mathrm{C}_{6} \mathrm{H}_{4}$ & 35 & 90 \\
\hline 11 & $4 k$ & $\mathrm{Me}$ & $4-\mathrm{CH}_{3} \mathrm{C}_{6} \mathrm{H}_{4}$ & 35 & 93 \\
\hline 12 & 41 & $\mathrm{Me}$ & $3,4-\left(\mathrm{OCH}_{3}\right)_{2} \mathrm{C}_{6} \mathrm{H}_{3}$ & $35(280)^{\mathrm{b}}$ & $90(85)^{b}$ \\
\hline 13 & $4 \mathrm{~m}$ & Et & $2,4-\mathrm{Cl}_{2} \mathrm{C}_{6} \mathrm{H}_{3}$ & $15(180)^{\mathrm{b}}$ & $95(90)^{b}$ \\
\hline 14 & $4 n$ & Et & $4-\mathrm{ClC}_{6} \mathrm{H}_{4}$ & $20(185)^{\mathrm{b}}$ & $89(80)^{\mathrm{b}}$ \\
\hline 15 & 40 & Et & $2-\mathrm{FC}_{6} \mathrm{H}_{4}$ & 18 & 86 \\
\hline 16 & $4 p$ & Et & $4-\mathrm{BrC}_{6} \mathrm{H}_{4}$ & 25 & 85 \\
\hline 17 & $4 q$ & Et & $4-\mathrm{MeSC}_{6} \mathrm{H}_{4}$ & 22 & 85 \\
\hline 18 & $4 r$ & Et & $\mathrm{C}_{6} \mathrm{H}_{5}$ & 27 & 87 \\
\hline
\end{tabular}

${ }^{\text {a Isolated yield. }}$

${ }^{\mathrm{b}}$ Conventional conditions.

$\left(25,50,65\right.$, and $\left.80^{\circ} \mathrm{C}\right)$ under sonochemical conditions. The results of this study are summarized in Table 1 . It is evident from the results that ethylene glycol is the most effective solvent among the selected solvents, giving the highest yield (95\%) and lower reaction time (25 $\mathrm{min})$ under ultrasound irradiation (entry 1). For comparison, the preparation of 4a was carried out under conventional heating at reflux conditions which furnished the desired product at lower yield and much higher reaction time $(180 \mathrm{~min})$ (Table 1 , entry 1). The results revealed that the reaction induced by ultrasonic irradiations offered better result than the conventional condition (Figure 1).

Using the optimized reaction conditions various derivatives of indenopyrido[2,3-d]pyrimidines $\mathbf{4 a - r}$ (Scheme 1) were prepared in high to excellent yields (85-97\%) and lower reaction times (Table 2 ).

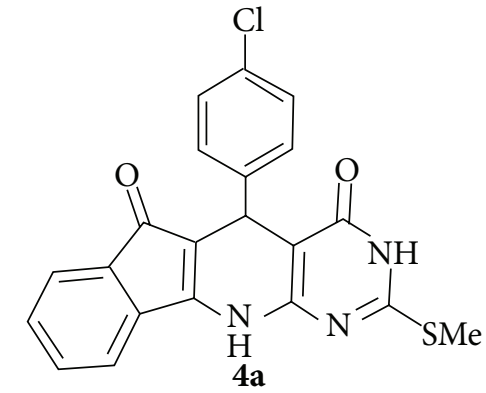

FIGURE 1

Due to remarkable results from the above experiments, we decided to broaden the scope of this protocol for 
TABLE 3: Synthesis of pyrimido[4,5-b]quinoline $\mathbf{6 a - f}$.

\begin{tabular}{lcccc}
\hline Entry & Product & $\mathrm{Ar}$ & Time $(\mathrm{min})$ & Yield $(\%)^{\mathrm{a}}$ \\
\hline 1 & $\mathbf{6 a}$ & $4-\mathrm{FC}_{6} \mathrm{H}_{4}$ & $10(180)^{\mathrm{b}}$ & $95(90)^{\mathrm{b}}$ \\
2 & $\mathbf{6 b}$ & 1 -naphtyl & $230)^{\mathrm{b}}$ & $93(87)^{\mathrm{b}}$ \\
3 & $\mathbf{6 c}$ & $4-\mathrm{CH}_{3} \mathrm{C}_{6} \mathrm{H}_{4}$ & $20(270)^{\mathrm{b}}$ & $89(82)^{\mathrm{b}}$ \\
4 & $\mathbf{6 d}$ & $4-\mathrm{CH}_{3} \mathrm{OC}_{6} \mathrm{H}_{4}$ & 23 & 87 \\
5 & $\mathbf{6 e}$ & 5-methylthiophen-2-yl & 25 & 82 \\
6 & $\mathbf{6 f}$ & $4-\mathrm{ClC}_{6} \mathrm{H}_{4}$ & 17 & 90 \\
\hline
\end{tabular}

${ }^{\mathrm{a}}$ Isolated yields.

${ }^{\mathrm{b}}$ Conventional conditions.

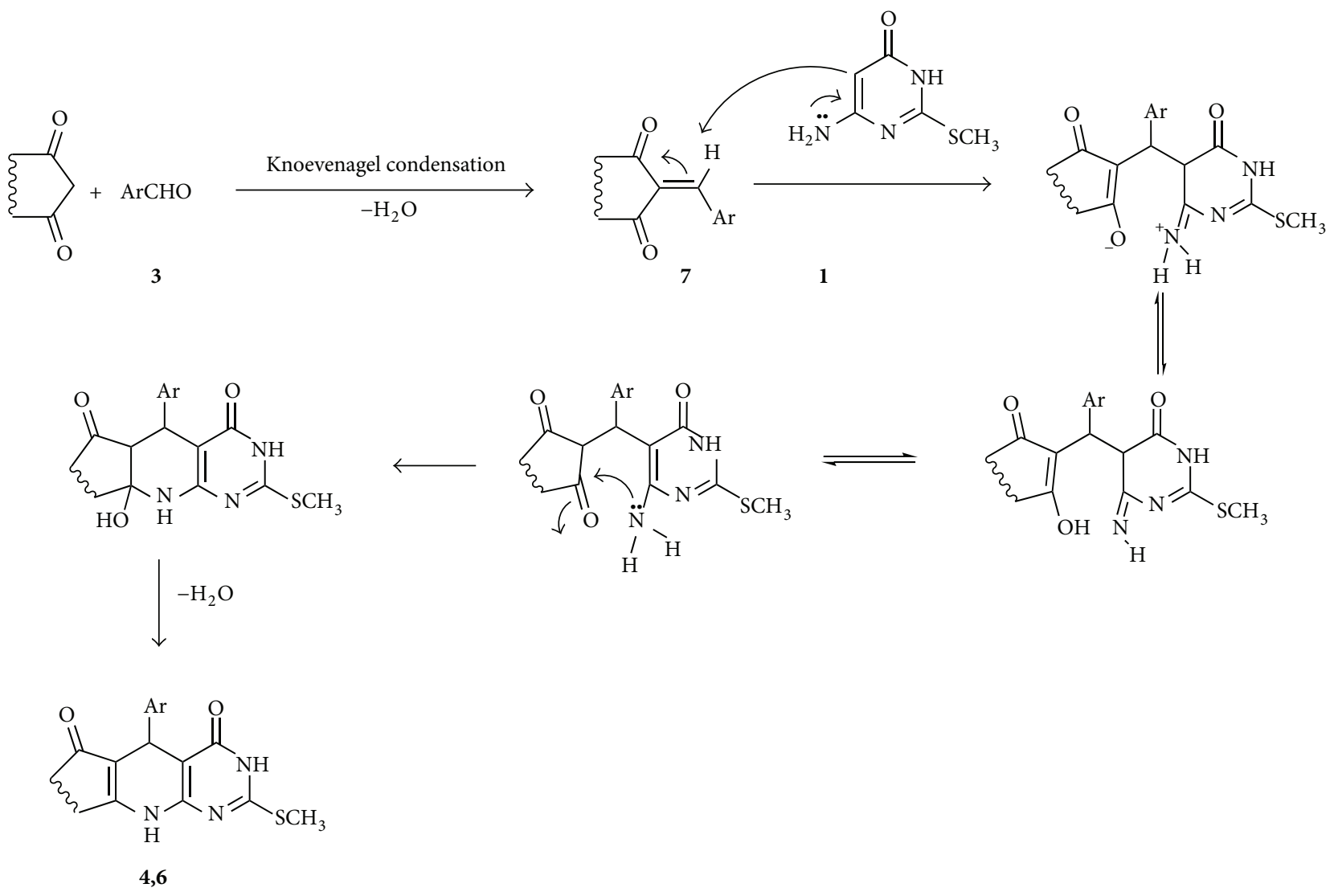

Scheme 3: The plausible mechanism of synthesis of fused pyrimidine derivatives $4 \mathbf{a}-\mathbf{r}$ and $\mathbf{6 a}-\mathbf{f}$.

the synthesis of quinoline derivatives as another fused pyrimidine derivative. We investigated the reaction between 6-amino-2-(ethylthio)pyrimidin-4(3H)-one 1, 1,3-cyclohexadione 5, and arylaldehydes 3 under the aforementioned optimized reaction conditions and obtained pyrimido[4,5b] quinoline-4,6-diones 6a-f (Scheme 2) in high yields (82$95 \%)$ and short reaction times (10-25 min) (Table 3).

The plausible mechanism of this MCRs involves Knoevenagel condensation between $\mathrm{CH}$-acid (2 or 5) and aryl aldehydes (3) resulting in the arylidene intermediate 7, followed by Michael addition of enaminone 1, cyclization, and removal of $\mathrm{H}_{2} \mathrm{O}$ to form the desired products (4 or 6) (Scheme 3).
All the synthesized pyridopyrimidine derivatives were characterized on the basis of elemental and spectral $\left({ }^{1} \mathrm{H}\right.$ NMR, ${ }^{13} \mathrm{C}$ NMR, and IR) analyses.

\section{Conclusion}

In summary, we have developed a simple, green, and efficient protocol for the synthesis of novel fused derivatives of pyrimidine under ultrasonic irradiations. The easy work of the products, without the use of catalyst, mild reaction condition, high to excellent yields, short reaction times, and cleaner reaction profiles are the notable features of this 
protocol. The method is amenable for the iterative generation of combinatorial libraries.

\section{Conflict of Interests}

The authors declare that there is no conflict of interests regarding the publication of this paper.

\section{Acknowledgments}

The authors are grateful to the Research Council of University of Guilan and Islamic Azad University, Rasht Branch, for financial support of this work.

\section{References}

[1] S. A. Giller, R. A. Zhuk, and M. I. U. Lidak, "Analogs of pyrimidine nucleosides. I. $\mathrm{N}_{1}$-(alpha-furanidyl) derivatives of natural pyrimidine bases and their antimetabolities," Doklady Akademii nauk SSSR, vol. 176, no. 2, pp. 332-335, 1967.

[2] A. R. Curreri, F. J. Ansfield, F. A. McIver, H. A. Waisman, and C. Hedelberger, "Clinical studies with 5-fluorouracil," Cancer Research, vol. 18, no. 4, pp. 478-484, 1958.

[3] N. K. Chaudhary, B. J. Montag, and C. Heidelberger, "Studies on fluorinated pyrimidines: III. The metabolism of 5-fluorouracil2- $\mathrm{C}^{14}$ and 5-fluoroorotic-2-C $\mathrm{C}^{14}$ acid in vivo," Cancer research, vol. 18 , no. 3, pp. 318-328, 1958.

[4] V. Malik, P. Singh, and S. Kumar, "Unique chlorine effect in regioselective one-pot synthesis of 1-alkyl-/allyl-3-(ochlorobenzyl) uracils: anti-HIV activity of selected uracil derivatives," Tetrahedron, vol. 62, no. 25, pp. 5944-5951, 2006.

[5] A. Holý, I. Votruba, M. Masojídková et al., "6-[2-(Phosphonomethoxy)alkoxy] pyrimidines with antiviral activity," Journal of Medicinal Chemistry, vol. 45, no. 9, pp. 1918-1929, 2002.

[6] P. Andres and A. Marhold, "A new synthesis of 5-trifluoromethyluracil," Journal of Fluorine Chemistry, vol. 77, no. 1, pp. 93-95, 1996.

[7] J. J. Reddick, S. Saha, J.-M. Lee, J. S. Melnick, J. Perkins, and T. P. Begley, "The mechanism of action of bacimethrin, a naturally occurring thiamin antimetabolite," Bioorganic and Medicinal Chemistry Letters, vol. 11, no. 17, pp. 2245-2248, 2001.

[8] B. Gauthier, "On the thiamines with an open thiazole ring. study of $O, S$-diacetylthiamine," Annales Pharmaceutiques Françaises, vol. 21, pp. 655-666, 1963.

[9] E. Wyrzykiewicz, G. Bartkowiak, Z. Nowakowska, and B. Kedzia, "Synthesis and antimicrobial properties of S-substituted derivatives of 2-thiouracil," Farmaco, vol. 48, no. 7, pp. 979-988, 1993.

[10] P. Sharma, N. Rane, and V. K. Gurram, "Synthesis and QSAR studies of pyrimido[4,5-d]pyrimidine-2,5-dione derivatives as potential antimicrobial agents," Bioorganic and Medicinal Chemistry Letters, vol. 14, no. 16, pp. 4185-4190, 2004.

[11] Y. M. Elkholy and M. A. Morsy, "Facile synthesis of 5, 6, 7, 8tetrahydropyrimido [4,5-b]-quinoline derivatives," Molecules, vol. 11, no. 11, pp. 890-903, 2006.

[12] B. S. Holla, M. Mahalinga, M. S. Karthikeyan, P. M. Akberali, and N. S. Shetty, "Synthesis of some novel pyrazolo[3,4d]pyrimidine derivatives as potential antimicrobial agents," Bioorganic and Medicinal Chemistry, vol. 14, no. 6, pp. 20402047, 2006.
[13] N. Ingarsal, G. Saravanan, P. Amutha, and S. Nagarajan, "Synthesis, in vitro antibacterial and antifungal evaluations of 2amino-4-(1-naphthyl)-6-arylpyrimidines," European Journal of Medicinal Chemistry, vol. 42, no. 4, pp. 517-520, 2007.

[14] X. L. Zhao, Y. F. Zhao, S. C. Guo, H. S. Song, D. Wang, and P. Gong, "Synthesis and anti-tumor activities of novel $[1,2,4]$ triazolo[1,5-a] pyrimidines," Molecules, vol. 12 , no. 5, pp. 1136-1146, 2007.

[15] L. Cordeu, E. Cubedo, E. Bandrés et al., "Biological profile of new apoptotic agents based on 2,4-pyrido[2,3-d]pyrimidine derivatives," Bioorganic and Medicinal Chemistry, vol. 15, no. 4, pp. 1659-1669, 2007.

[16] N. M. Khalifa, N. S. Ismail, and M. M. Abdulla, "Synthesis and reactions of fused cyanopyrimidine derivatives and related glycosides as antianginal and cardio protective agents," Egyptian Pharmaceutical Journal, vol. 4, pp. 277-288, 2005.

[17] R. L. Smith, R. J. Barrett, and E. Sanders-Bush, "Neurochemical and behavioral evidence that quipazine-ketanserin discrimination is mediated by serotonin 2A receptor," Journal of Pharmacology and Experimental Therapeutics, vol. 275, no. 2, pp. 1050-1057, 1995.

[18] F. Awouters, J. Vermeire, F. Smeyers et al., "Oral antiallergic activity in ascaris hypersensitive dogs: a study of known antihistamines and of the new compounds ramastine (R 57 959) and levocabastine (R 50 547)," Drug Development Research, vol. 8, no. 1-4, pp. 95-102, 1986.

[19] Y. Yanagihara, H. Kasai, T. Kawashima, and T. Shida, "Immunopharmacological studies on TBX, a new antiallergic drug (1). Inhibitory effects on passsive cutaneous anaphylaxis in rats and guinea pigs," The Japanese Journal of Pharmacology, vol. 48, no. 1, pp. 91-101, 1988.

[20] R. D. Larsen, E. G. Corley, A. O. King et al., "Practical route to a new class of LTD4 receptor antagonists," The Journal of Organic Chemistry, vol. 61, no. 10, pp. 3398-3405, 1996.

[21] Y.-L. Chen, K.-C. Fang, J.-Y. Sheu, S.-L. Hsu, and C.-C. Tzeng, "Synthesis and antibacterial evaluation of certain quinolone derivatives," Journal of Medicinal Chemistry, vol. 44, no. 14, pp. 2374-2377, 2001.

[22] G. Roma, M. Di Braccio, G. Grossi, F. Mattioli, and M. Ghia, "1,8 -Naphthyridines IV. 9-Substituted N, N -dialkyl-5-(alkylamino or cycloalkylamino) $[1,2,4]$ triazolo $[4,3-$ a] $[1,8]$ naphthyridine6-carboxamides, new compounds with anti-aggressive and potent anti-inflammatory activities," European Journal of Medicinal Chemistry, vol. 35, no. 11, pp. 1021-1035, 2000.

[23] D. Dubé, M. Blouin, C. Brideau et al., "Quinolines as potent 5lipoxygenase inhibitors: synthesis and biological profile of L746,530," Bioorganic \& Medicinal Chemistry Letters, vol. 8, no. 10, pp. 1255-1260, 1998.

[24] M. P. Maguire, K. R. Sheets, K. McVety, A. P. Spada, and A. Zilberstein, "A new series of PDGF receptor tyrosine kinase inhibitors: 3-Substituted quinoline derivatives," Journal of Medicinal Chemistry, vol. 37, no. 14, pp. 2129-2137, 1994.

[25] O. Billker, V. Lindo, M. Panico et al., "Identification of xanthurenic acid as the putative inducer of malaria development in the mosquito," Nature, vol. 392, no. 6673, pp. 289-292, 1998.

[26] T. K. Bradshaw and D. W. Hutchinson, "5-Substituted pyrimidine nucleosides and nucleotides," Chemical Society Reviews, vol. 6, no. 1, pp. 43-62, 1977.

[27] T. Sasaki, K. Minamoto, T. Suzuki, and S. Yamashita, "Search for a simpler synthetic model system for intramolecular 1,3dipolar cycloaddition to the 5,6-double bond of a pyrimidine nucleoside," Tetrahedron, vol. 36, no. 7, pp. 865-870, 1980. 
[28] R. Marumoto and Y. Furukawa, "Kondensierte pyrimidine. III. Synthese von Isoxazolo [3, 4-d] pyrimidinen," Chemical and Pharmaceutical Bulletin, vol. 25, no. 11, pp. 2974-2982, 1977.

[29] H. Griengl, E. Wanek, W. Schwarz, W. Streicher, B. Rosenwirth, and E. De Clercq, " 2 '-Fluorinated arabinonucleosides of 5(2-haloalkyl)uracil: synthesis and antiviral activity," Journal of Medicinal Chemistry, vol. 30, no. 7, pp. 1199-1204, 1987.

[30] G. Shaw, Comprehensive Heterocyclic Chemistry, Volume 3, A. R. Katritzky, C.W. Rees, Eds., p. 57, Pergamon, Oxford, UK, 1984.

[31] A. Agarwal and P. M. S. Chauhan, "Solid supported synthesis of structurally diverse dihydropyrido[2,3-d] pyrimidines using microwave irradiation," Tetrahedron Letters, vol. 46, no. 8, pp. 1345-1348, 2005.

[32] G. H. Mahdavinia, S. Rostamizadeh, A. M. Amani, and Z. Emdadi, "Ultrasound-promoted greener synthesis of aryl14-H-dibenzo[a,j]xanthenes catalyzed by $\mathrm{NH}_{4} \mathrm{H}_{2} \mathrm{PO}_{4} / \mathrm{SiO}_{2}$ in water," Ultrasonics Sonochemistry, vol. 16, no. 1, pp. 7-10, 2009.

[33] G. Cravotto and P. Cintas, "Power ultrasound in organic synthesis: moving cavitational chemistry from academia to innovative and large-scale applications," Chemical Society Reviews, vol. 35, no. 2, pp. 180-196, 2006.

[34] T. J. Mason and J. P. Lorimer, Applied Sonochemistry: The Uses of Power Ultrasound in Chemistry and Processing, Wiley-VCH, 2002.

[35] D. Mantu, C. Moldoveanu, A. Nicolescu, C. Deleanu, and I. I. Mangalagiu, "A facile synthesis of pyridazinone derivatives under ultrasonic irradiation," Ultrasonics Sonochemistry, vol. 16, no. 4, pp. 452-454, 2009.

[36] J. T. Li, Y. Yin, L. Li, and M. X. Sun, "A convenient and efficient protocol for the synthesis of 5-aryl-1,3-diphenylpyrazole catalyzed by hydrochloric acid under ultrasound irradiation," Ultrasonics Sonochemistry, vol. 17, no. 1, pp. 11-13, 2010.

[37] L. Pizzuti, P. L. G. Martins, B. A. Ribeiro et al., "Efficient sonochemical synthesis of novel 3,5-diaryl-4,5-dihydro- $1 \mathrm{H}$ pyrazole-1-carboximidamides," Ultrasonics Sonochemistry, vol. 17, no. 1, pp. 34-37, 2010.

[38] C.-L. Ni, X.-H. Song, H. Yan, X.-Q. Song, and R.-G. Zhong, "Improved synthesis of diethyl 2,6-dimethyl-4-aryl-4H-pyran3,5-dicarboxylate under ultrasound irradiation," Ultrasonics Sonochemistry, vol. 17, no. 2, pp. 367-369, 2010.

[39] A. Bazgir, S. Ahadi, R. Ghahremanzadeh, H. R. Khavasi, and P. Mirzaei, "Ultrasound-assisted one-pot, three-component synthesis of spiro[indoline-3, $4^{\prime}$-pyrazolo[3,4-b]pyridine]-2,6' $\left(1^{\prime} H\right)$-diones in water," Ultrasonics Sonochemistry, vol. 17, no. 2 , pp. 447-452, 2010.

[40] Z.-H. Zhang, J.-J. Li, and T.-S. Li, "Ultrasound-assisted synthesis of pyrroles catalyzed by zirconium chloride under solvent-free conditions," Ultrasonics Sonochemistry, vol. 15, no. 5, pp. 673676, 2008.

[41] A. R. Khosropour, "Ultrasound-promoted greener synthesis of 2,4,5-trisubstituted imidazoles catalyzed by $\mathrm{Zr}(\mathrm{acac})_{4}$ under ambient conditions," Ultrasonics Sonochemistry, vol. 15, no. 5, pp. 659-664, 2008.

[42] R. H. Nia, M. Mamaghani, F. Shirini, and K. Tabatabaeian, "A convenient one-pot three-component approach for regioselective synthesis of novel substituted pyrazolo[1,5-a]pyrimidines using $\mathrm{Fe}^{+3}$-montmorillonite as efficient catalyst," Journal of Heterocyclic Chemistry, vol. 51, no. 2, pp. 363-367, 2014.

[43] M. Mamaghani, F. Shirini, N. O. Mahmoodi, A. AzimiRoshan, and A. Monfared, “on water' organic synthesis: three-component one-pot synthesis of novel bis(1(cyclohexylamino)-1-oxoalkyl or aryl) fumarates," Journal of the Iranian Chemical Society, vol. 11, no. 3, pp. 659-664, 2014.

[44] M. Mamaghani, F. Shirini, N. O. Mahmoodi, A. Azimi-Roshan, and H. Hashemlou, "A green, efficient and recyclable Fe ${ }^{+3} @ \mathrm{~K} 10$ catalyst for the synthesis of bioactive pyrazolo[3,4-b]pyridin$6(7 \mathrm{H})$-ones under "on water" conditions," Journal of Molecular Structure, vol. 1051, pp. 169-176, 2013.

[45] R. Hossein Nia, M. Mamaghani, K. Tabatabaeian, F. Shirini, and M. Rassa, "An expeditious regioselective synthesis of novel bioactive indole-substituted chromene derivatives via one-pot three-component reaction," Bioorganic and Medicinal Chemistry Letters, vol. 22, no. 18, pp. 5956-5960, 2012.

[46] M. Mamaghani, A. Loghmanifar, and M. R. Taati, "An efficient one-pot synthesis of new 2-imino-1,3-thiazolidin-4-ones under ultrasonic conditions," Ultrasonics Sonochemistry, vol. 18, no. 1, pp. 45-48, 2011.

[47] M. Nikpassand, M. Mamaghani, F. Shirini, and K. Tabatabaeian, "A convenient ultrasound-promoted regioselective synthesis of fused polycyclic 4-aryl-3-methyl-4,7-dihydro- $1 H$-pyrazolo[3,4b]pyridines," Ultrasonics Sonochemistry, vol. 17, no. 2, pp. 301305, 2010.

[48] M. Mamaghani, N. O. Mahmoodi, F. Shirini, A. Azimi-Roshan, and A. Monfared, "On water sonochemical multicomponent synthesis of novel bioactive $1,1^{\prime}$-(Aryl)bis(2-(cyclohexylamino)2-oxoethane-1,1-diyl) Di(alkanoates and benzoates)," Journal of Chemistry, vol. 2013, Article ID 125959, 8 pages, 2013.

[49] P. Crepaldi, B. Cacciari, M.-C. Bonache et al., "6-Amino-2mercapto-3H-pyrimidin-4-one derivatives as new candidates for the antagonism at the $\mathrm{P}_{2} \mathrm{Y}_{12}$ receptors," Bioorganic \& Medicinal Chemistry, vol. 17, no. 13, pp. 4612-4621, 2009. 

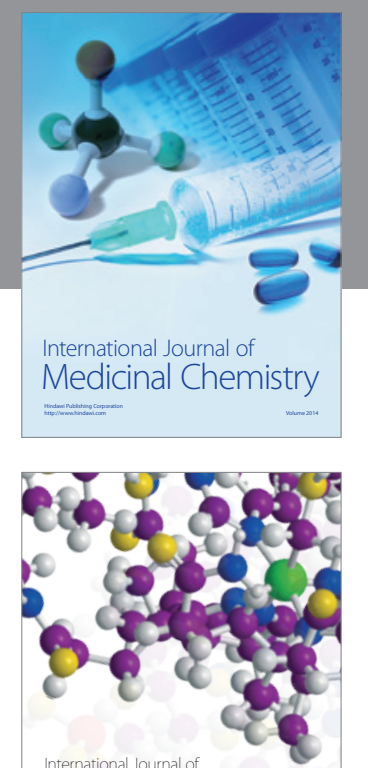

\section{Carbohydrate} Chemistry

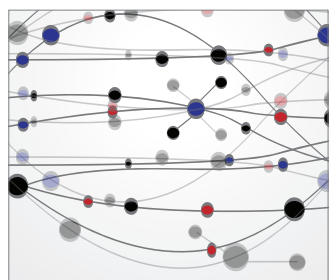

The Scientific World Journal
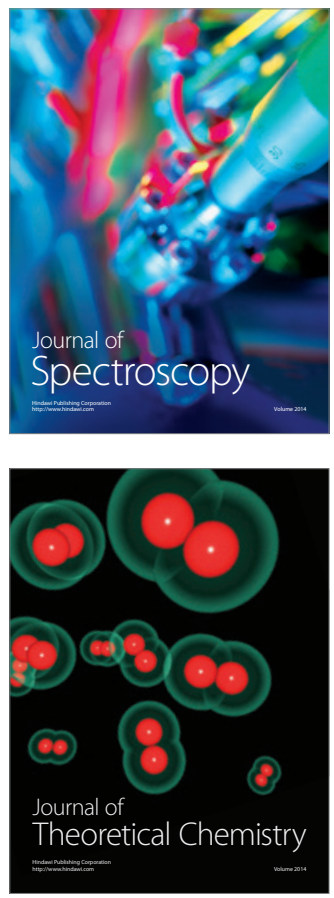
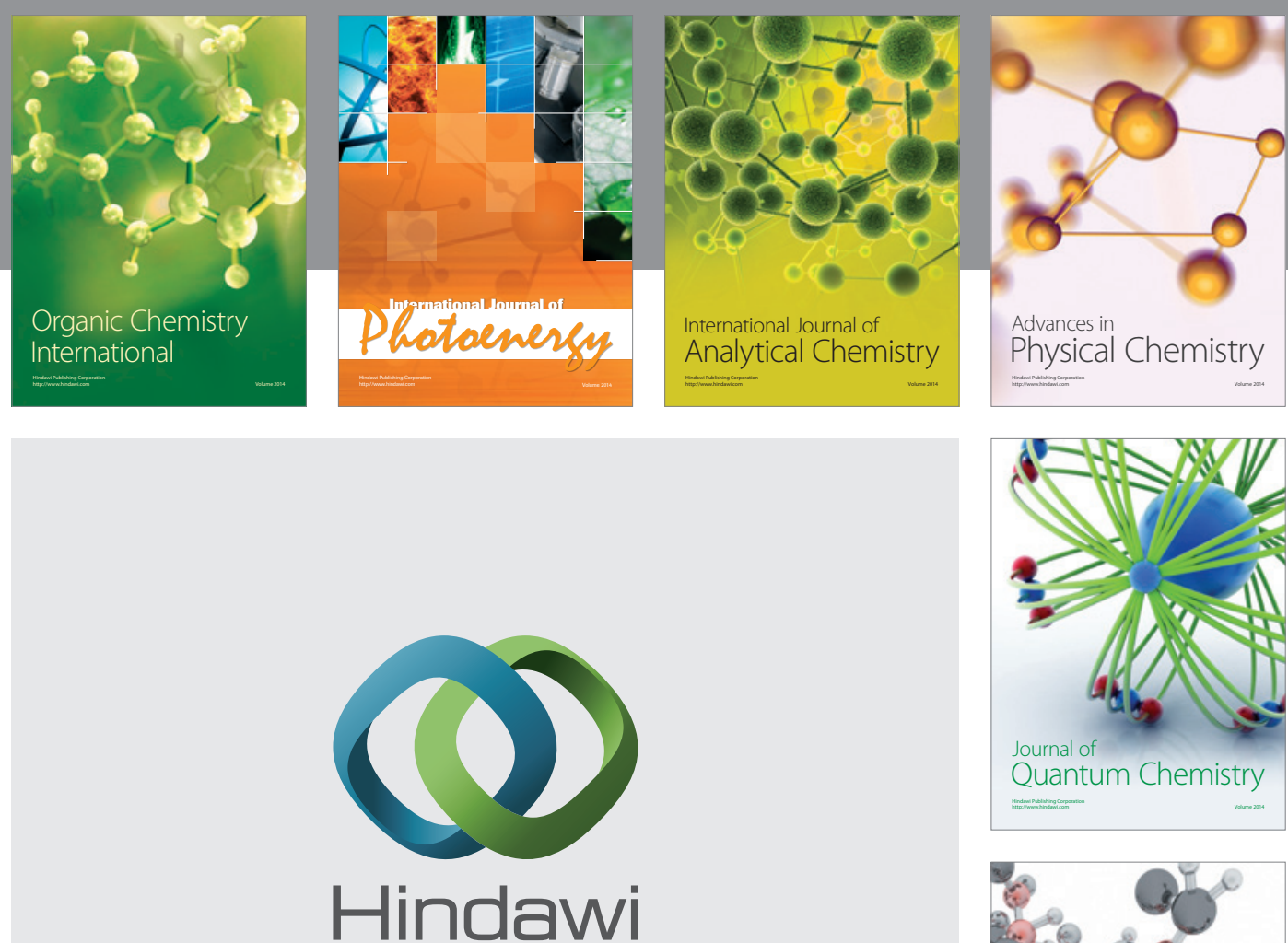

Submit your manuscripts at

http://www.hindawi.com

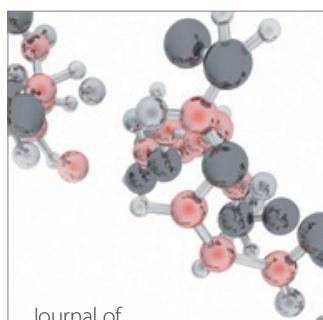

Analytical Methods

in Chemistry

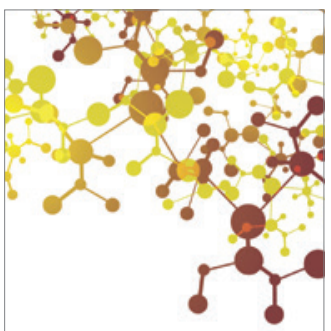

Journal of

Applied Chemistry

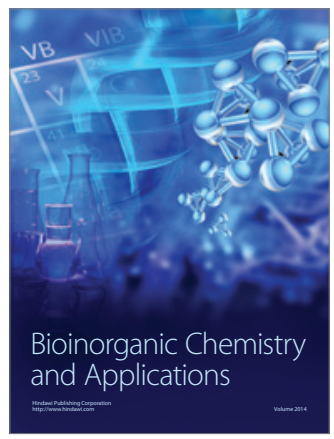

Inorganic Chemistry
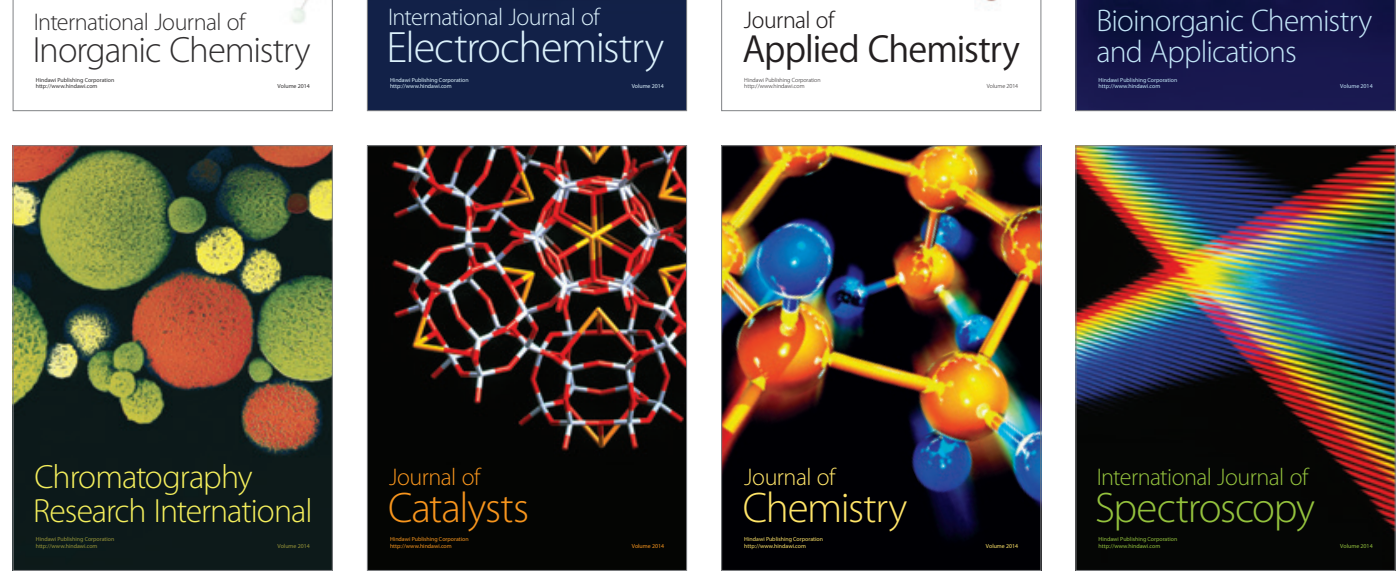